\title{
MEASURING DETAILED CHEMICAL ABUNDANCES FROM CO-ADDED MEDIUM-RESOLUTION SPECTRA. I. TESTS USING MILKY WAY DWARF SPHEROIDAL GALAXIES AND GLOBULAR CLUSTERS*
}

\author{
Lei Yang ${ }^{1}$, Evan N. Kirby ${ }^{2,6}$, Puragra Guhathakurta ${ }^{3}$, Eric W. Peng ${ }^{1,4}$, and Lucy Cheng ${ }^{5}$ \\ ${ }^{1}$ Department of Astronomy, Peking University, 5 Yiheyuan Road, Haidian, Beijing 100871, China; yangleiseti@gmail.com \\ ${ }^{2}$ Department of Astronomy, California Institute of Technology, Mail Stop 249-17, Pasadena, CA 91125, USA \\ ${ }^{3}$ University of California Observatories/Lick Observatory, Department of Astronomy \& Astrophysics, University of California, \\ 1156 High Street, Santa Cruz, CA 95064, USA \\ ${ }^{4}$ Kavli Institute for Astronomy and Astrophysics, Peking University, 5 Yiheyuan Road, Haidian, Beijing 100871, China \\ 5 The Harker School, 500 Saratoga Avenue, San Jose, CA 95129, USA \\ Received 2012 July 7; accepted 2013 March 3; published 2013 April 8
}

\begin{abstract}
The ability to measure metallicities and $\alpha$-element abundances in individual red giant branch (RGB) stars using medium-resolution spectra $(R \approx 6000)$ is a valuable tool for deciphering the nature of Milky Way dwarf satellites and the history of the Galactic halo. Extending such studies to more distant systems like Andromeda is beyond the ability of the current generation of telescopes, but by co-adding the spectra of similar stars, we can attain the necessary signal-to-noise ratio $(\mathrm{S} / \mathrm{N})$ to make detailed abundance measurements. In this paper, we present a method to determine metallicities and $\alpha$-element abundances using the co-addition of medium-resolution spectra. We test the method of spectral co-addition using high-S/N spectra of more than 1300 RGB stars from Milky Way globular clusters and dwarf spheroidal galaxies obtained with the Keck II telescope/DEIMOS spectrograph. We group similar stars using photometric criteria and compare the weighted ensemble average abundances $([\mathrm{Fe} / \mathrm{H}]$, $[\mathrm{Mg} / \mathrm{Fe}],[\mathrm{Si} / \mathrm{Fe}],[\mathrm{Ca} / \mathrm{Fe}]$, and $[\mathrm{Ti} / \mathrm{Fe}])$ of individual stars in each group with the measurements made on the corresponding co-added spectrum. We find a high level of agreement between the two methods, which permits us to apply this co-added spectra technique to more distant RGB stars, like stars in the M31 satellite galaxies. This paper outlines our spectral co-addition and abundance measurement methodology and describes the potential biases in making these measurements.
\end{abstract}

Key words: galaxies: abundances - galaxies: dwarf - galaxies: evolution - galaxies: stellar content - Local Group

Online-only material: color figures

\section{INTRODUCTION}

The Local Group is dominated by the Milky Way and Andromeda galaxies, with large families of dwarf satellite galaxies. The accessibility of these fantastic targets, particularly the dwarf spheroidals (dSphs) around the Milky Way, enables us to trace the dynamical and chemical properties of individual stars in order to investigate their formation. For decades, evidence has been growing that much of the material in the universe condensed into small dark matter halos at an early stage, and over a Hubble time, many of these halos contribute to the growth of massive galaxies in a "chaotic accretion" (Searle \& Zinn 1978; White \& Rees 1978; Cole et al. 2000; Diemand et al. 2007). From observations, Helmi et al. (2006) showed that there were metal-poor stars in the Milky Way halo with $[\mathrm{Fe} / \mathrm{H}]<-3.0$ dex, which did not seem to exist in dSph galaxies, and therefore ruled out present-day dSphs as the building blocks of the Milky Way halo. Recently, however, Kirby et al. (2008a, hereafter KGS08, 2009, 2010) determined the iron abundance distribution of vast majority of red giant branch (RGB) stars in globular clusters (GCs) and ultra-faint $\mathrm{dSphs}$, and they found a significant metal-poor tail of stars does exist in the Milky Way satellites, supporting the hierarchical formation of the stellar halo.

\footnotetext{
* Data herein were obtained at the W. M. Keck Observatory, which is operated as a scientific partnership among the California Institute of Technology, the University of California, and NASA. The Observatory was made possible by the generous financial support of the W. M. Keck Foundation.

6 Hubble Fellow.
}

To investigate the formation of big spiral galaxies, especially their stellar halo accretion history, chemical abundance patterns obtained from individual stars are crucial indicators (Wheeler et al. 1989; Worthey 1994; Mannucci et al. 2010). Stars produce a diversity of elements through nucleosynthesis, which are dispersed into the interstellar medium, and are then mixed with material in subsequent star formation. Generally, iron-peak elements, such as vanadium and iron, are mainly generated by Type Ia supernovae (SNe Ia; Tinsley 1980) which are important contributors to the total iron fraction in galaxies (Greggio \& Renzini 1983). Correspondingly, the $\alpha$-elements, like oxygen, magnesium, silicon, calcium, titanium, etc., are produced in core-collapse supernovae (SNe II) whose progenitors are massive stars with typical stellar masses greater than $9 M_{\odot}$ (Wheeler et al. 1989; Woosley \& Weaver 1995; Gilmore 2004). Furthermore, compared to SNe II, which have a timescale of around 10 Myr (Pagel 1997; Woosley \& Janka 2005), SNe Ia have a longer timescale of at least $\sim 1$ Gyr (Matteucci \& Recchi 2001; Ishigaki et al. 2012). Hence, a plot of $[\alpha / \mathrm{Fe}]$ versus $[\mathrm{Fe} / \mathrm{H}]$ tells us about the relative contribution of SNe Ia and SNe II in star formation and evolution of a stellar system as a function of time, and can be used as a clock to measure the intensity of star formation at early stages. Additionally, comparing the distribution of metallicity and $\alpha$-element abundances between the different stellar components of the Milky Way and its companions is a way of determining their evolutionary relationships.

Photometry is commonly used to determine the metallicities of old RGB stars. This technique uses the locations of RGB stars in the color-magnitude diagram (CMD) and compares them to empirical relations (Armandroff et al. 1993), fitting functions 
(Saviane et al. 2000), or theoretical stellar tracks (e.g., Harris \& Harris 2000; Mouhcine et al. 2005; Lianou et al. 2010) to derive photometric estimates of the metallicity. The metallicities of distant stars in the Andromeda system have also been estimated in this way. Da Costa et al. (1996, 2000, 2002) used HST/ WFPC2 to measure photometric metallicities and obtained age estimates using the RGB and horizontal branch in three dSph galaxies, Andromeda I, Andromeda II, and Andromeda III, around M31. Kalirai et al. (2010) studied hundreds of RGB stars in six M31 dSphs, producing a luminosity-metallicity relation for dwarfs and compared it with that of their Milky Way counterparts. However, photometric metallicity estimates are often not as accurate as spectroscopic metallicity for two main reasons. First, the $\alpha$ abundance of star often has to be assumed. Second, the degeneracy of age and metallicity (Worthey 1994), even with resolved CMDs, is difficult to untangle. Lianou et al. (2011) compared photometric metallicity and spectroscopic metallicity based on the near-infrared $\mathrm{Ca}$ triplet analysis of RGBs in five Galactic dSphs, and they found that the agreement is good for metallicities between $-2.0 \mathrm{dex}$ and $-1.5 \mathrm{dex}$ but that a high fraction of intermediate-age stars would produce unreliable values.

Deriving chemical abundances using spectroscopy is much more reliable, but also expensive in terms of data collection. A stellar spectrum contains a wealth of information, allowing us to derive important stellar parameters like effective temperature, surface gravity, metallicity, and other heavy elements abundances. Several empirical calibrations can be employed to measure metallicity. Popular methods include Ca II K $\lambda 3933$ (Preston 1961; Zinn \& West 1984; Beers et al. 1999) and the Ca near-infrared triplet calibration (Bica \& Alloin 1987; Armandroff \& Zinn 1988; Olszewski et al. 1991; Rutledge et al. 1997; Foster et al. 2010) with the prerequisite that $[\mathrm{Ca} / \mathrm{Fe}]$ must be assumed. Unfortunately, these calibrations fail when $[\mathrm{Fe} / \mathrm{H}]<-2.2$ (Kirby et al. 2008b).

The most reliable way to measure abundances is to use highresolution spectroscopy (HRS; $R \gtrsim 20,000$ ). This technique has been used to analyze the detailed chemical abundance distribution of the Milky Way system and its satellites (Shetrone et al. 1998, 2001, 2003; Venn et al. 2004; Tolstoy et al. 2009; Letarte et al. 2010). HRS measurements of individual stars beyond the Milky Way and its satellites, however, are extremely challenging. Moreover, HRS is difficult to multiplex and often requires observing one star at a time. For these reasons, mediumresolution spectroscopy $(R \approx 6000)$, for which abundances of some elements can still be well measured, is an optimal choice for large sample stars in Milky Way satellites, and even for the M31 system (Guhathakurta et al. 2006; Koch et al. 2007).

Previous studies have targeted stars in the Milky Way satellites (e.g., Lanfranchi \& Matteucci 2004; Shetrone et al. 2009; Strigari et al. 2010). The largest sample was given by KGS08 and Kirby et al. $(2009,2010)$ who observed more than 2500 RGB stars in Milky Way GCs and dSphs at medium resolution using Keck/DEIMOS (Faber et al. 2003). They performed a multi-element abundance analysis using pixel-to-pixel matching based on a grid of synthetic stellar spectra. They verified their abundance measurement technique by comparing their results with the abundances derived from high-resolution spectra. In their study, in addition to finding a long metal-poor tail in ultra-faint dSphs, matching that of the Galactic halo, they derived trends for individual $\alpha$-elements $(\mathrm{Mg}, \mathrm{Si}, \mathrm{Ca}, \mathrm{Ti})$ versus metallicity, demonstrating that there was not much metal enrichment before the onset of SNe I (Kirby et al. 2011a, 2011b).
Observations of M31's halo present a more complex accretion history (Ibata et al. 2001; Choi et al. 2002; Reitzel \& Guhathakurta 2002; McConnachie et al. 2004; Guhathakurta et al. 2006, 2010; Kalirai et al. 2009, 2010; Collins et al. 2010; Tanaka et al. 2010), indicating that a study of abundance trends in its satellites could be more interesting and challenging. However, the larger distance and fainter apparent magnitudes hamper any detailed investigation of individual stellar abundances for dwarf satellites in M31, although there have been attempts to obtain detailed chemical abundance patterns in M31 GCs using high-resolution, integrated-light spectroscopy (Colucci et al. 2009). One way to address this problem is by co-adding many similar stars to produce spectra with higher signal-to-noise ratio $(\mathrm{S} / \mathrm{N})$. This process, if done properly, would extend our ability to obtain elemental abundance analysis to larger distances, but there is also the potential for introducing biases.

The co-addition of spectra to measure the ensemble properties of similar objects has a long history in spectral processing. Adelman \& Leckrone (1985) used co-addition for the ultraviolet and optical region of spectrum. Holberg et al. (2003) co-added multiple observations of individual white dwarf stars to enhance the $\mathrm{S} / \mathrm{N}$ and combined them into a single spectrum. Gallazzi et al. (2008) used co-added spectra of galaxies with similar velocity dispersions, absolute $r$-band magnitude, and $4000 \AA$ break values for those regions of parameter space where individual spectra had lower S/N. Most recently, Schlaufman et al. (2011, 2012) compared the average metallicities and $\alpha$-element abundances between the elements of cold halo substructure and the kinematically smooth stellar inner halo along lines of sight in the Sloan Extension for Galactic Understanding and Exploration (SEGUE) by co-adding spectra of the metalpoor main-sequence turnoff stars identified by the SEGUE Stellar Parameter Pipeline. Using noise-degraded spectra, they found that the mean square error (MSE) of abundances derived from co-added spectra is from 0.05 dex for metal-rich (iron-rich) stars to 0.2 dex for $[\mathrm{Fe} / \mathrm{H}]$ and $[\alpha / \mathrm{Fe}]$ for the most metal-poor stars. In a test using the GCs M13 and M15, the MSE roughly equals to 0.1 dex for most of the metal-rich stars and to 0.2 dex for metal-poor stars, in both $[\mathrm{Fe} / \mathrm{H}]$ and $[\alpha / \mathrm{Fe}]$. However, the individual abundances of $\alpha$-elements are inaccessible in their measurements. In this work, we use weighted spectral coaddition of RGB stars that share similar intrinsic properties to increase the $\mathrm{S} / \mathrm{N}$ so that individual $\alpha$-elements can be measured.

All data used in this paper are from the same data sets used in KGS08 and Kirby et al. (2009, 2010), which involve thousands RGB stars in Milky Way GCs and dSphs. We test our co-addition method by measuring metallicity $([\mathrm{Fe} / \mathrm{H}])$ and four $\alpha$-element abundances ([Mg/Fe], [Si/Fe], [Ca/Fe], [Ti/Fe]). We used the same definition of chemical abundances as defined in KGS08. ${ }^{7}$

First, we present a short summary of the observations and data reduction in Section 2. In Section 3, we describe the method of abundance determination using a synthetic spectral grid, as well as our method of co-addition: star selection and grouping, weighted co-adding, and abundance measurement. In Section 4, we present the comparison of weighted ensemble average of individual abundances to abundances measured from co-added spectra. We also discuss discrepancies and biases between the two results. We summarize our work in Section 5.

\footnotetext{
7 In their work, $12+\log [n(\mathrm{Fe}) / n(\mathrm{H})]_{\odot}=7.52$ is adopted in Section 4 of KGS08, where $n$ is number density (as adopted by Sneden et al. 1992). The abundances of other elements adopted solar composition from Anders \& Grevesse (1989) in Table 8 of Kirby et al. (2010).
} 


\section{OBSERVATION AND DATA REDUCTION}

All the medium-resolution spectra of RGB stars used in this study are from KGS08 and Kirby et al. (2009, 2010), which include 2947 RGB stars in 8 Milky Way dSph galaxies and 654 RGB stars in 14 Galactic GCs. The observations were performed with DEIMOS on the Keck II telescope. The spectrograph configuration used the OG550 filter with the 1200 line $\mathrm{mm}^{-1}$ grating at a central wavelength of $\sim 7800 \AA$ with a slit width of 0 . 7 . The spectral resolution is $\sim 1.2 \AA$ to $\sim 1.3 \AA$ (corresponding to a resolving power $6500<R<7000$ at $8500 \AA$ ) with a spectral range of 6300-9100 A. Exposures of $\mathrm{Kr}, \mathrm{Ne}, \mathrm{Ar}$, and $\mathrm{Xe}$ arc lamps were used for wavelength calibration and exposure of a quartz lamp provided the flat field. The DEIMOS data reduction pipeline developed by the DEEP galaxy redshift survey ${ }^{8}$ was used to extract one-dimensional spectra (Newman et al. 2012; Cooper et al. 2012). The pipeline traced the edges of slits in the flat field to determine the CCD location of each slit. A polynomial fit to the CCD pixel locations of arc lamp lines provided the wavelength solution. Each exposure of stellar targets was rectified and then sky subtracted based on a B-spline model of the night sky emission lines. Then, the exposures were combined with cosmic ray rejection into one two-dimensional spectrum for each slit. Finally, the one-dimensional stellar spectrum was extracted from a small spatial window encompassing the light of star in the two-dimensional spectrum. The product of the pipeline was a wavelength calibrated, sky-subtracted, cosmic-ray-cleaned, one-dimensional spectrum for each target. A hot star template spectrum was employed to remove the terrestrial atmospheric absorption introduced into the stellar spectra. In continuum determination, a B-spline was used to fit the "continuum regions" 9 of the spectra. Each pixel was weighted by its inverse variance in the fit, and the fit was performed iteratively such that pixels that deviated from the fit by more than $5 \sigma$ were removed from the next iteration of the fit. For further details, please see KGS08. Table 1 lists all the stellar systems used in this study.

\section{ABUNDANCE MEASUREMENTS}

Kirby et al. presented a technique for multi-element abundance measurements of medium-resolution spectra, which enable them to determine individual $\alpha$-element abundances of RGB stars in the Milky Way GCs and dwarf satellite galaxies. In this technique a large grid of synthetic spectra is used, so there is no restriction imposed on the metallicity range, overcoming the problems encountered by other methods. In brief, the photometric effective temperature ( $\left.T_{\text {eff }}\right)$ and surface gravity ( $\log g$ ) are determined from isochrone fitting on the CMD using three different model isochrones (Kirby et al. 2009)-Yonsei-Yale (Demarque et al. 2004), Victoria-Regina (VandenBerg et al. 2006), and Padova (Girardi et al. 2002) — and an empirical colorbased $T_{\text {eff }}$ (Ramírez \& Meléndez 2005). Then, they adopted the Levenberg-Marquardt algorithm (mpf it, written by Markwardt 2009) to find the best-fitting synthetic spectrum to the observed spectrum in several iterative steps by minimizing the $\chi^{2}$ calculated from the degraded synthetic spectrum and the observed spectrum. Lastly, the stellar parameters of the best-fitting synthetic spectrum are presented as the observed ones. With dif-

\footnotetext{
8 http://astro.berkeley.edu/ cooper/deep/spec $2 \mathrm{~d} /$. The analysis pipeline used to reduce the DEIMOS data was developed at UC Berkeley with support from NSF grant AST-0071048.

9 They call spectral regions with synthetic flux greater than 0.96 and a minimum width of $0.5 \AA$ "continuum regions."
}

Table 1

RGB Spectral Data Sets

\begin{tabular}{|c|c|c|}
\hline \multirow[t]{2}{*}{ Name } & \multicolumn{2}{|c|}{ Number of RGB Stars } \\
\hline & Total & $\log g \leqslant 1.4$ \\
\hline \multicolumn{3}{|l|}{ Globular clusters } \\
\hline NGC 6205 (M13) & 68 & 9 \\
\hline NGC 7078 (M15) & 134 & 26 \\
\hline NGC 1904 (M79) & 58 & 17 \\
\hline NGC 2419 & 95 & 40 \\
\hline NGC 7006 & 11 & 9 \\
\hline NGC 7492 & 20 & 5 \\
\hline NGC $5024($ M53) & 49 & 2 \\
\hline NGC $6656(\mathrm{M} 22)^{\mathrm{a}}$ & 50 & 1 \\
\hline NGC $5053^{\mathrm{a}}$ & 49 & 1 \\
\hline NGC $288^{a}$ & 30 & 0 \\
\hline NGC 5904 (M5) & 51 & 6 \\
\hline NGC $6838($ M71) & 34 & 1 \\
\hline NGC 7089 (M2) & 44 & 5 \\
\hline Pal $13^{\mathrm{a}}$ & 10 & 0 \\
\hline \multicolumn{3}{|l|}{ dSphs } \\
\hline Canes Venatici I & 174 & 36 \\
\hline Draco & 298 & 38 \\
\hline Fornax & 675 & 280 \\
\hline Leo I & 813 & 571 \\
\hline Leo II & 258 & 119 \\
\hline Sculptor & 376 & 153 \\
\hline Sextans & 141 & 16 \\
\hline Ursa Minor & 212 & 21 \\
\hline
\end{tabular}

ferent elemental masks, the abundances measured include iron abundance $([\mathrm{Fe} / \mathrm{H}])$ and four $\alpha$-elements $(\mathrm{Mg}, \mathrm{Si}, \mathrm{Ca}$, and $\mathrm{Ti})$. The four individual $\alpha$-elements were measured by considering only spectral regions most sensitive to the corresponding element. For example, the $\mathrm{Mg}$ wavelength mask covers about 20 neutral Mg lines in the DEIMOS spectral range. Even though all of the $\alpha$-elements vary together in the synthetic spectrum, only $\mathrm{Mg}$ lines are used to determine $[\mathrm{Mg} / \mathrm{Fe}]$. For those are interested in elemental masks, $\mathrm{KGSO}^{10}$ and Kirby et al. (2009) ${ }^{11}$ depicted more details on the construction of the wavelength masks. They performed extensive comparisons of their medium-resolution results with HRS elemental abundances from previous studies to validate their technique. In this study, we inherit the idea of Kirby's technique and make proper modification to meet our demands to determine chemical abundances of more distant RGB stars beyond the Milky Way. But, firstly, we aim to test our coadded spectra method with the medium-resolution spectra used by Kirby et al., and the membership of these RGB stars has been confirmed by Kirby et al.

\subsection{Synthetic Spectral Library}

We accomplished all chemical abundance analysis on the basis of a large grid of synthetic spectra generated by Kirby et al. (KGS08, 2009, 2010). Based on ATLAS9 model atmospheres (Kurucz 1993; Sbordone et al. 2004; Sbordone 2005) without convective overshooting (Castelli et al. 1997; Castelli \& Kurucz 2004; Castelli 2005), and a line list of atomic and

\footnotetext{
10 Spectral mask, Table 2.

11 Section 4.6 and Figure 5.
} 
Table 2

Atmospheric Parameter Grid

\begin{tabular}{lcccr}
\hline \hline Quantity & Minimum & Maximum & Step & Number \\
\hline$T_{\text {eff }}(\mathrm{K})$ & 3500 & 8000 & $100\left(T_{\text {eff }} \leqslant 5600\right)$ & 22 \\
& & & $200\left(T_{\text {eff }} \geqslant 5600\right)$ & 12 \\
$\log g\left(\mathrm{~cm} \mathrm{~s}^{-2}\right), T_{\text {eff }} \leqslant 6800$ & 0.0 & 5.0 & 0.5 & 11 \\
$\log g\left(\mathrm{~cm} \mathrm{~s}^{-2}\right), T_{\text {eff }} \geqslant 7000$ & 0.5 & 5.0 & 0.5 & 10 \\
{$[\mathrm{M} / \mathrm{H}]($ atmosphere $)$} & -4.0 & 0.0 & 0.5 & 9 \\
{$[\mathrm{M} / \mathrm{H}]$ (spectra) } & -4.0 & 0.0 & 0.1 & 41 \\
{$[\alpha / \mathrm{Fe}]$} & -0.8 & +1.2 & 0.1 & 21 \\
\hline
\end{tabular}

molecular transition data from the Vienna Atomic Line Database (Kupka et al. 1999), Kirby et al. synthesized spectra using the local equilibrium, plane-parallel spectrum synthesis code MOOG (Sneden 1973), which span the same wavelength range as the data (6300-9100 $\AA$ ) with a resolution of $0.02 \AA$. The synthetic spectra fail in modeling of the Ca II triplet, $\mathrm{Mg} \mathrm{I} \lambda 8807$, and the absorption lines of TiO. To avoid an unexpected discontinuity they recomputed new opacity distribution functions for the new grid with DFSYNTHE code (Castelli 2005) and employed the solar composition of Anders \& Grevesse (1989), but for Fe they used Sneden et al. (1992) (Kirby et al. 2009). To prevent unwittingly discarding the extremely metal-poor stars beyond the preliminary boundary of grid, they also expanded the synthetic spectral grid limit to $[\mathrm{Fe} / \mathrm{H}]=-5.0$ (Kirby et al. $2010)$. The value of $[\alpha / \mathrm{Fe}]$ for the stellar model atmospheres would be different for each individual $\alpha$-elements because elements have been measured only with the spectral regions most sensitive to the corresponding element. Therefore, an additional subgrid with the extra dimension of $\alpha$-element abundance $\left([\alpha / \mathrm{Fe}]_{\mathrm{abund}}\right)$ is also generated for more accurate measurement of $[\mathrm{Mg} / \mathrm{Fe}],[\mathrm{Si} / \mathrm{Fe}],[\mathrm{Ca} / \mathrm{Fe}]$, and $[\mathrm{Ti} / \mathrm{Fe}]$ at fixed $[\alpha / \mathrm{Fe}]_{\mathrm{atm}}$. This spectral library includes four dimensions: effective temperature $\left(T_{\text {eff }}\right)$, surface gravity $(\log g)$, metallicity $([\mathrm{Fe} / \mathrm{H}]), \alpha$ abundance $\left([\alpha / \mathrm{Fe}]_{\mathrm{atm}}\right)$ of the stellar atmosphere. Table 2 gives the limited ranges and steps of these five parameters. This spectral grid is available online, and readers with interests in this grid are recommended to refer to more details in Kirby (2011).

\subsection{Individual Stellar Abundances}

To determine stellar abundances, we developed an independent code based on Kirby et al.'s technique with some refinements for the application to co-added spectra. For testing purposes, most of the medium-resolution spectra used have $\mathrm{S} / \mathrm{N}>20 \mathrm{pixel}^{-1}$ with mean S/N around $80 \mathrm{pixel}^{-1}$.

In spectral co-addition, an essential step is to rebin the spectra in preparation for co-adding. Our approach rebins each science spectrum onto a common wavelength region (6300-9100 A with step $0.25 \AA$ ). The same has been done to the degraded synthetic spectrum which is going to be compared with the rebinned science one. Considering our co-added spectra approach aims to be applied on the RGB stars of M31 dwarf satellite galaxies whose spectroscopic temperatures are not available, we fixed the effective temperature, as well as the surface gravity, with the value derived from photometry. When we measured the effective temperature and surface gravity, the Yonsei-Yale isochrone fitting was carried out on the CMD at an assumed age of $14 \mathrm{Gyr}$ and $[\alpha / \mathrm{Fe}]=+0.3$ for all RGB stars; however, in Kirby et al.'s work they only estimated $\log g$ by photometry. After setting the initial parameters, we performed the abundance determination on the rebinned spectra. In order to verify that our method works well on individual stars, we redetermined chemical abundances of all RGB stars with our code. Figure 1 shows the comparison of $[\mathrm{Fe} / \mathrm{H}]$ between Kirby's and ours. The stars in the Figure 1 are also used for later co-addition test, but some stars whose spectra had insufficient $\mathrm{S} / \mathrm{N}$ to measure a particular element have been removed. The selection detailed is discussed in Section 3.3.

\subsection{Surface Gravity Restriction}

To start, we performed chemical abundance determination for more than $3600 \mathrm{RGB}$ stars from $14 \mathrm{GCs}$ and $8 \mathrm{dSph}$ galaxies. The stellar ages of these RGB stars are difficult to estimate but fortunately have only a small impact on the measured chemical abundances (Harris et al. 1999; Frayn \& Gilmore 2002; Lianou et al. 2010), so we assumed an age of $14 \mathrm{Gyr}$ for all RGB stars (Grebel \& Gallagher 2004) and set $[\alpha / \mathrm{Fe}]=+0.3$ empirically. Then, the effective temperature $\left(T_{\text {eff }}\right)$ and surface gravity $(\log g)$ of member stars were estimated by fitting YonseiYale isochrones on the CMD. We then proceeded to measure the individual abundances as described in Section 3.2. We found some element abundances of some stars were unmeasurable, and we expected that low $\mathrm{S} / \mathrm{N}$ is a possible reason. Thus, for the purposes of this test, stars for which we cannot measure a particular element abundance were not used in the coaddition. Additionally, previous observations of RGB stars in M31 showed that only stars with $M_{I} \leqslant-2.5$ are accessible for spectroscopy. So we further introduced a cut in $\log g$. Given the roughly linear relationship between $M_{I}$ and $T_{\text {eff }}$, this corresponds to a selection in $\log g$. Figure 2 shows the linear relationship between photometric $\log g$ and absolute magnitude in the $I$-band $\left(M_{I}\right)$ for seven dSphs (except Fornax, for which we do not have $I$-band data). From Figure 2, $M_{I}=-2.5$ roughly corresponds to a cut at $\log g=1.40$. Thus, we only selected stars having photometric $\log g \leqslant 1.40$ for the co-addition. The number of stars left for each dSph and GC after this selection is listed in Table 3. Only eight GCs have enough stars for the following test.

\subsection{Grouping and Co-addition}

We consider photometric effective temperature $\left(T_{\text {eff }}\right)$ and the photometric metallicity estimate $\left([\mathrm{Fe} / \mathrm{H}]_{\text {phot }}\right)$, respectively, to organize the remaining stars into groups for co-adding. The photometric metallicities are also derived by YonseiYale theoretical isochrones fitting with an age of $14 \mathrm{Gyr}$ (see Section 3.3). We used a cut at $\log g \leqslant 1.40$ to ensure that all stars lie in a range of about $1 \mathrm{dex}$ in $\log g$. Moreover, the synthetic spectral measurements use neutral metal lines only which are nearly insensitive to surface gravity. Therefore, $\log g$ barely changes the strength of spectral features, making it acceptable not to include $\log g$ in the binning. 


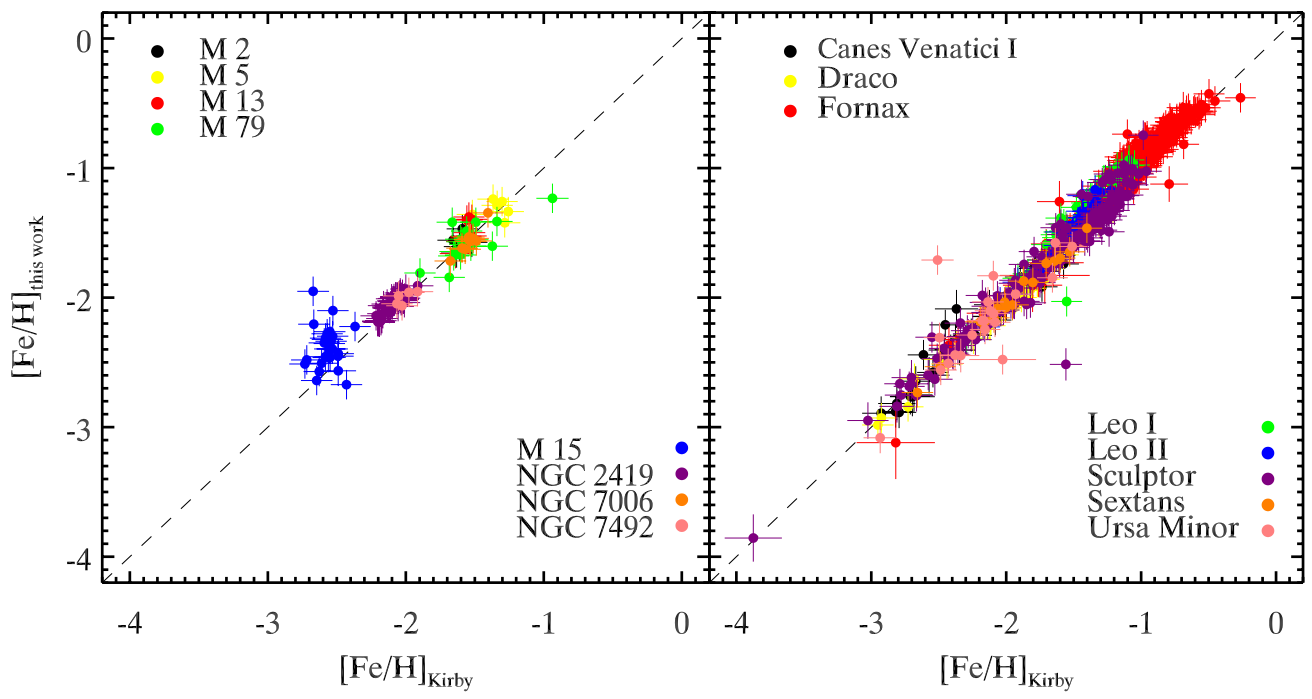

Figure 1. Comparison of metallicity measured from medium-resolution spectra for individual stars between Kirby et al. and our codes, developed for co-added spectra. The left panel presents results for eight GCs and the right panel for eight dSphs. All these RGB stars meet the $\log g \leqslant 1.4$ threshold. The key difference between these two measurements for individual stars is in our codes we used a fixed $T_{\text {eff }}$ and $\log g$ derived from photometric estimates vs. allowing $T_{\text {eff }}$ to float during the spectral fitting in Kirby et al.'s codes. The Levenberg-Marquardt algorithm is adopted to find the best-fitting spectrum with several iterative steps then give the abundance. The error bars shown include both the random errors in the fit and the systematic errors adopted from Kirby et al.

(A color version of this figure is available in the online journal.)

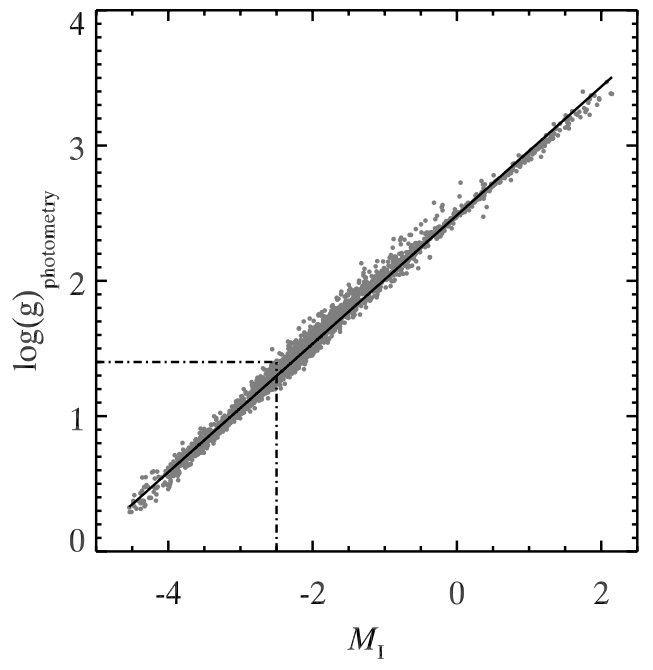

Figure 2. We use a linear relationship between photometric surface gravity $(\log g)$ and $I$-band absolute magnitude $\left(M_{I}\right)$, which is converted from apparent magnitude with the extinction-corrected distance modulus, to select stars whose $\log g \leqslant 1.40\left(\left(M_{I}\right) \geqslant-2.5\right)$. The plot contains all dSph RGB stars except for Fornax for which we have no $I$-band data. The line is the best-fit linear relation. Stars with $\log g \leqslant 1.4$ are used to produce co-added spectra.

The goal of this study is, for each grouping, to compare the weighted-average abundances of the individual stars (the input) with the abundances measured on the co-added spectra (the output). For this purpose, it is important that each star used has a measurable abundance. Our pipeline is able to measure $[\mathrm{Fe} / \mathrm{H}]$ in all sample stars, but for some of them, individual $\alpha$-elements (e.g., $\mathrm{Mg}$ ) may not be measurable due to the quality of the spectra. Including these spectra in the grouping would bias the measurement on the co-added spectrum (but contribute nothing to the weighted average), so we are very careful to construct separate groupings for each element measured, i.e., a co-added spectrum for testing $[\mathrm{Mg} / \mathrm{Fe}]$ consists only of stars that have reliable $[\mathrm{Mg} / \mathrm{Fe}]$ measurements individually. This allows us to
Table 3

Star Groups

\begin{tabular}{lcc}
\hline \hline Name of Galaxies & $N_{\text {bin }}{ }^{\mathrm{a}}$ & $N_{\text {good stars }} \mathrm{b}^{\circ}$ \\
\hline & Globular clusters & \\
\hline NGC 6205(M13) & 1 & 9 \\
NGC 7078(M15) & 4 & 5 \\
NGC 1904(M79) & 3 & 5 \\
NGC 2419 & 5 & 5 \\
NGC 7006 & 1 & 9 \\
NGC 7492 & 1 & 5 \\
NGC 5904(M5) & 1 & 6 \\
NGC 7089(M2) & 1 & 5 \\
\hline & $\mathrm{d}$ & \\
\hline Canes Venatici I & 4 & 5 \\
Draco & 6 & 5 \\
Fornax & 30 & 8 \\
Leo I & 44 & 8 \\
Leo II & 11 & 8 \\
Sculptor & 16 & 5 \\
Sextans & 2 & 5 \\
Ursa Minor & 2 &
\end{tabular}

Notes.

${ }^{\text {a }} N_{\text {bin }}$ is the number of bins for each stellar system.

${ }^{\text {b }} N_{\text {good stars }}$ is the minimum number of good stars we set for each bin.

use the maximum number of available stars for testing each element.

After ranking member stars by their $\left(T_{\text {eff }}\right)_{\text {phot }}$ and $[\mathrm{Fe} / \mathrm{H}]_{\text {phot }}$ separately, we make sure that each group contains at least five stars for which all five elemental abundances are measurable individually. We set 8 as the minimum number of stars for Fornax, Leo II, and Sculptor, and 10 for Leo I for their large number of stars. Table 3 lists details for each GC and dSph. When we co-add spectra together for one bin, we produce five different coadds; that is, for each elemental abundance of $[\mathrm{Fe} / \mathrm{H}],[\mathrm{Mg} / \mathrm{Fe}]$, 


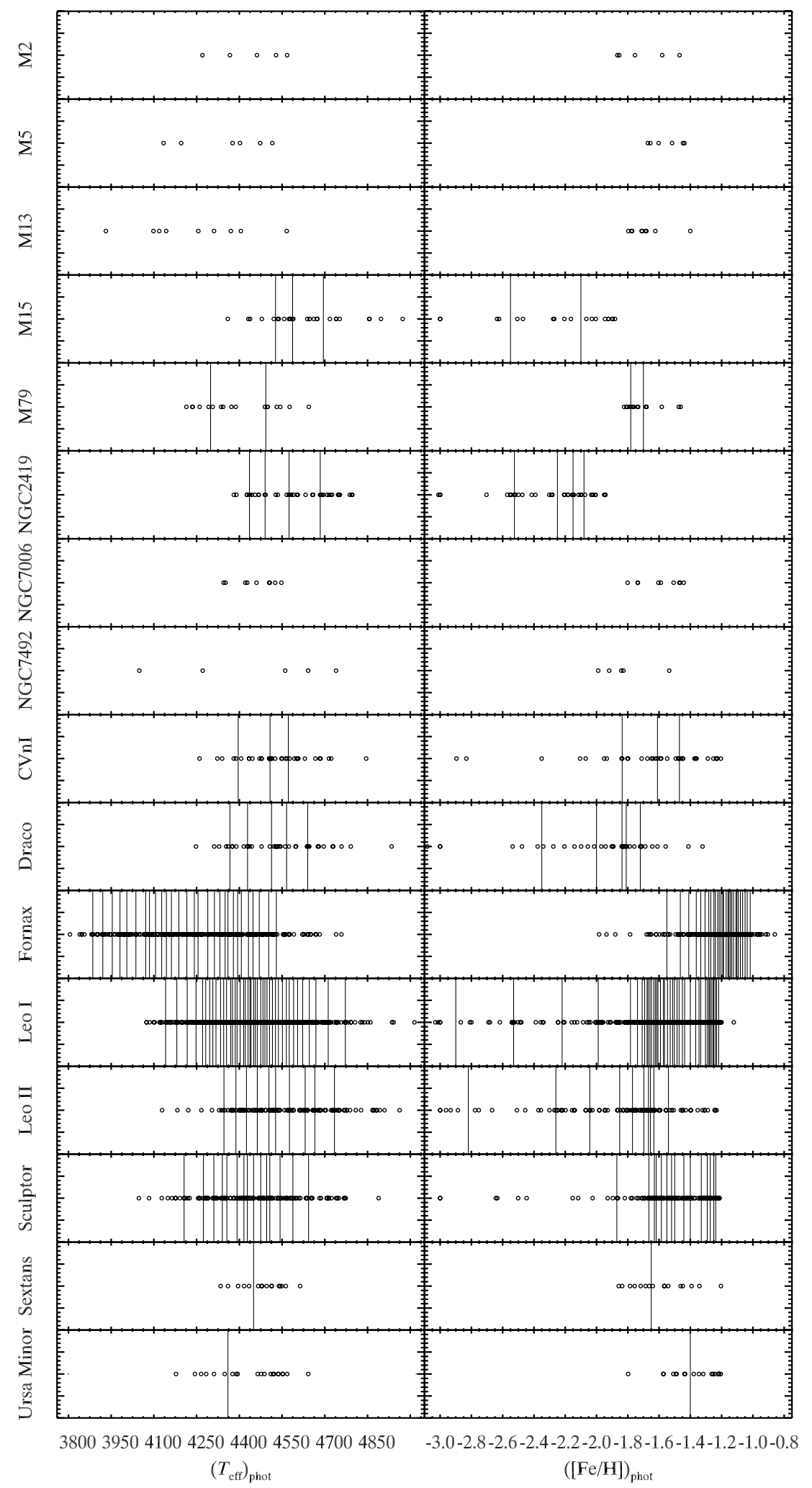

Figure 3. Two scenarios have been used to bin stars. The open circles are individual stars used in this test. The vertical lines split the test stars into groups by two ways. Left panels show stars binned by photometric effective temperature. Right panels show stars binned by photometric metallicity. There are five GCs (M2, M5, M13, NGC 7006, and NGC 7492) that have one bin for the limited number of stars. From this comparison, the dSphs, which are most affected by the two scenarios, are Fornax, Leo I, and Sculptor. We detail the impact of different binning schemes in the discussion section.

$[\mathrm{Si} / \mathrm{Fe}],[\mathrm{Ca} / \mathrm{Fe}]$, and $[\mathrm{Ti} / \mathrm{Fe}]$, we only add the spectra whose elemental abundance is measurable individually, and use that co-added spectrum to determine the corresponding elemental abundance. The bad spectral regions therefore make no con- tribution to the co-added spectrum for the element of interest, which is equivalent to the elemental abundance derived from the weighted ensemble average. Figure 3 shows two binning scenarios in detail for eight GCs and eight dSphs, by photo- 

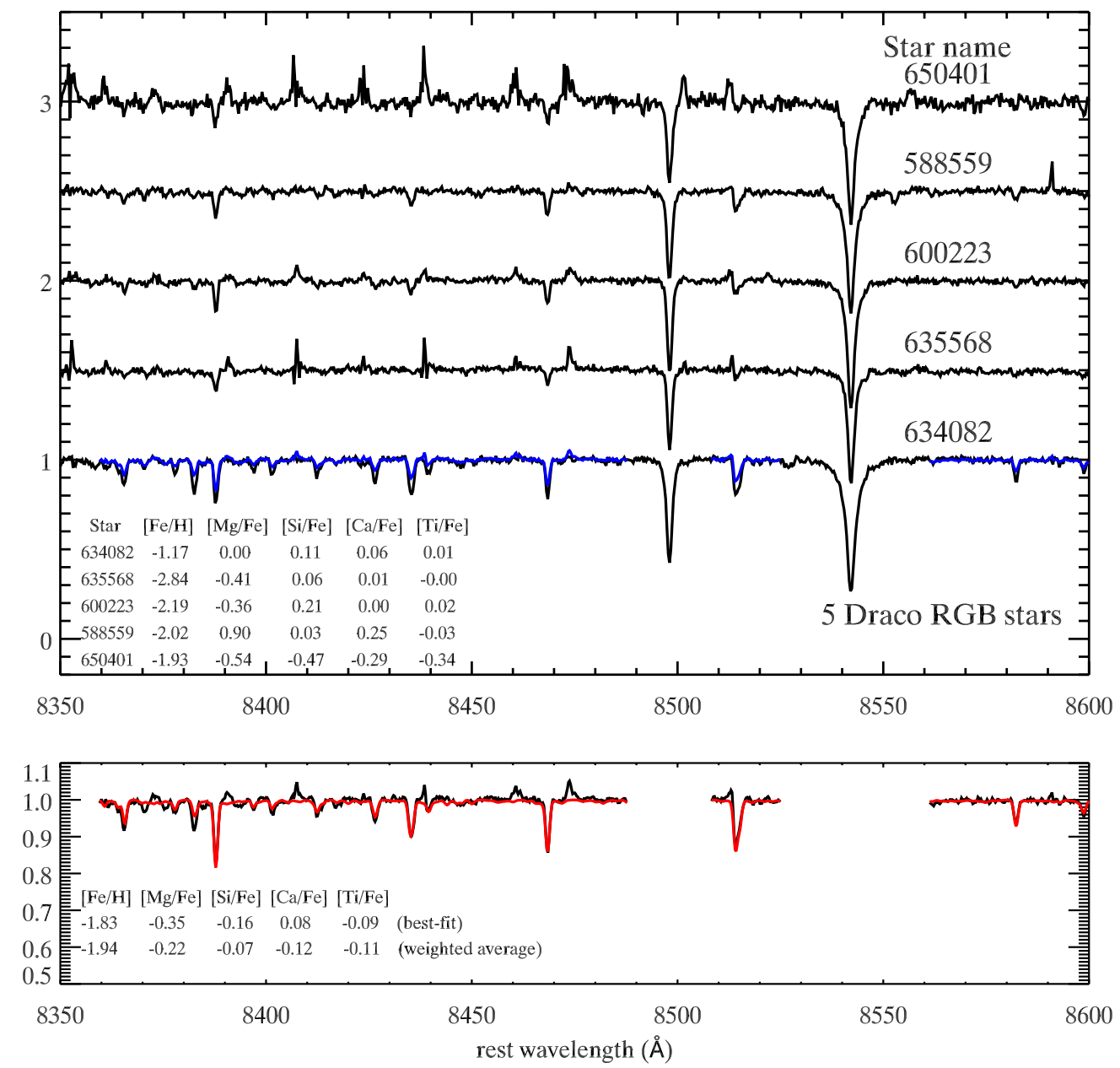

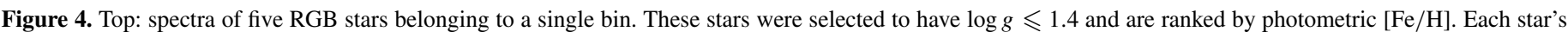

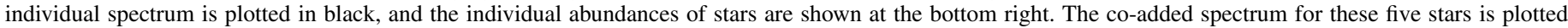

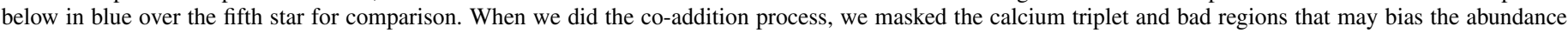

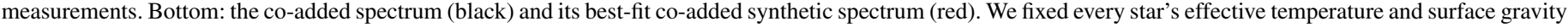

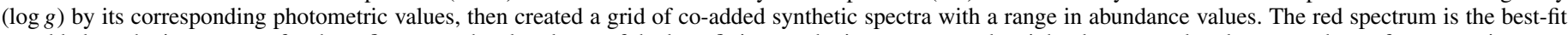
co-added synthetic spectrum for these five stars; the abundance of the best-fitting synthetic spectrum and weighted-average abundance are shown for comparison.

(A color version of this figure is available in the online journal.)

metric effective temperature and metallicity. As the left panels show, stars having expanded distribution in $T_{\text {eff }}$ are binned more evenly, especially for Fornax, Leo I, and Sculptor, whose $[\mathrm{Fe} / \mathrm{H}]_{\text {phot }}$ are more concentrated. The impacts of this difference to the measured metallicities for these bins are clearly shown in the later figures.

For the observed spectrum, we use pixel masks to remove bad spectral regions, like telluric absorption and cosmic rays, before rebinning. Keck/DEIMOS has eight CCD and the whole spectrum spans two CCDs, so we exclude 5 pixels near the end of each CCD which may cause artifacts. We then rebin the spectrum onto a common wavelength range (6300-9100 $\AA$ ) and add fluxes of the normalized rebinned spectra together weighted by the rebinned inverse variance. The co-addition equation is

$$
\bar{x}_{\text {pixel }, i}=\frac{\sum_{j=1}^{n}\left(x_{\text {pixel }, i j} / \sigma_{\text {pixel }, i j}^{2}\right)}{\sum_{j=1}^{n} 1 / \sigma_{\text {pixel }, i j}^{2}},
$$

where $x_{\text {pixel, } i j}$ represents the flux in the $i$ th pixel of the $j$ th spectrum in a group of stars, $\sigma_{\text {pixel }, i j}^{2}$ is the variance of $x_{\text {pixel }, i j}, n$ represents the total number of spectra in the group, $\bar{x}_{\text {pixel }, i}$ is the weighted-average flux of the $i$ th pixel of $n$ spectra.
For consistency, we also create a grid of co-added synthetic spectra. First, for one group of stars, we pick the same number of synthetic spectra with same chemical abundances but with different $T_{\text {eff }}$ and $\log g$. The synthetic spectra are chosen to have the $T_{\text {eff }}$ and $\log g$ as determined by the photometric estimates of the observed stars. Second, we smoothed all the synthetic spectra with a Gaussian filter to match the spectral resolution science spectra. Then, we co-add the synthetic spectra in the same way as the science spectra (Equation (1)), with each pixel of the synthetic spectrum having the same weight as the corresponding pixel in the science spectrum.

Figure 4 presents an example of a co-added observed spectrum (blue in the top panel and black in the bottom panel and its best-fit co-added synthetic spectrum (red in the bottom panel). Stars with similar stellar properties should have the most similar spectra which are then used to determine chemical abundances and stellar parameters. When we carried out the co-addition, we also avoided the calcium triplet, which failed in spectra synthesis. When the comparison was executed, only the spectral regions determined to be most sensitive to the element were considered in the abundance determination, as showed in the bottom panel of Figure 4. 


\section{5. $[\mathrm{Fe} / \mathrm{H}]$ and $[\alpha / \mathrm{Fe}]$ Determination}

Following KGS08 and Kirby et al. (2009), we determine individual chemical abundances with elemental "masks" that only cover the wavelength regions that are sensitive to that particular element. Each element, therefore, has its own mask, but for $[\alpha / \mathrm{Fe}]_{\mathrm{atm}}$ the mask is a combination of the four $\alpha$-element masks. We adopt the Levenberg-Marquardt algorithm implemented in IDL programs written by Markwardt (2009) to find the best match to the co-added observed spectra. This algorithm finds the best co-added synthetic spectrum by minimizing $\chi^{2}$ of pixels between the co-added observed spectrum and the co-added synthetic spectrum over iterations. The detailed procedure of our abundance determination is described in 11 steps.

1. $[\mathrm{Fe} / \mathrm{H}]$, first pass. For a bin of stars, the co-added observed spectrum is compared with the co-added synthetic spectra. In this pass, only the spectral regions that are sensitive to $\mathrm{Fe}$ absorption are considered. The $[\alpha / \mathrm{Fe}]_{\text {atm }}$ (total $\alpha$-element abundance) and $[\alpha / \mathrm{Fe}]_{\text {abund }}$ (abundance of individual element, $\mathrm{Mg}, \mathrm{Si}, \mathrm{Ca}, \mathrm{Ti})$ were fixed at 0 , while the $[\mathrm{Fe} / \mathrm{H}]$ value was varied until the best-fit synthetic spectrum was found. When individual synthetic spectra were chosen, the $T_{\text {eff }}$ and $\log g$ were set to be photometric values of individual science stars in that group and the abundances were set to be the $[\alpha / \mathrm{Fe}]_{\mathrm{atm}},[\alpha / \mathrm{Fe}]_{\text {abund }}$, and $[\mathrm{Fe} / \mathrm{H}]$ values. The derived $[\mathrm{Fe} / \mathrm{H}]$ of the best-fit synthetic spectrum was fixed and used in later steps.

2. $[\alpha / \mathrm{Fe}]_{\mathrm{atm}}$, first pass. All the variables were fixed except the total $\alpha$-element abundance, $[\alpha / \mathrm{Fe}]_{\mathrm{atm}}$.

3. Continuum refinement. To refine the continuum of the observed spectrum, the co-added science spectrum was divided by the co-added synthetic spectrum with the parameters determined in steps 1 and 2, resulting in a quotient spectrum without absorption lines. Then we used a B-spline with a breakpoint spacing of 250 pixels to fit the quotient. Finally, we divided the co-added observed spectrum by the spline fit. This forces a better continuum match between the observed and synthetic spectra.

4. Steps 1-3 were repeated until $[\mathrm{Fe} / \mathrm{H}]$ and $[\alpha / \mathrm{Fe}]_{\mathrm{atm}}$ changed by less than 0.001 dex between consecutive passes.

5. Sigma clipping. In this pass, we masked pixels whose absolute difference from the best-fit synthetic spectrum with the parameters determined in step 3 exceeded 2.5 times their standard deviations. Then we used this sigma-clipped spectrum to repeat step 1. All parameters except $[\mathrm{Fe} / \mathrm{H}]$ were fixed. The resulting $[\mathrm{Fe} / \mathrm{H}]$ value is the second pass value.

6. $[\alpha / \mathrm{Fe}]_{\mathrm{atm}}$, second pass. We repeated step 2 with the sigmaclipped spectrum and the $[\mathrm{Fe} / \mathrm{H}]$ determined from step 5 .

7. $[\mathrm{Fe} / \mathrm{H}]$, third pass. Step 5 was repeated with the value of $[\alpha / \mathrm{Fe}]_{\mathrm{atm}}$ determined from step 6 .

8. $[\mathrm{Mg} / \mathrm{Fe}]$. Only the spectral regions sensitive to $\mathrm{Mg}$ absorption lines were considered. We fixed $[\mathrm{Fe} / \mathrm{H}]$ and $[\alpha / \mathrm{Fe}]_{\mathrm{atm}}$ at the values attained from steps 7 and 6 , and varied $[\alpha / \mathrm{Fe}]_{\text {abund }}$ to measure $[\mathrm{Mg} / \mathrm{Fe}]$.

9. $[\mathrm{Si} / \mathrm{Fe}]$. We repeated step 8 but replaced $[\mathrm{Mg} / \mathrm{Fe}]$ with [Si/Fe].

10. $[\mathrm{Ca} / \mathrm{Fe}]$. We repeated step 8 but replaced $[\mathrm{Mg} / \mathrm{Fe}]$ with $[\mathrm{Ca} / \mathrm{Fe}]$.

11. $[\mathrm{Ti} / \mathrm{Fe}]$. We repeated step 8 but replaced $[\mathrm{Mg} / \mathrm{Fe}]$ with $[\mathrm{Ti} / \mathrm{Fe}]$.

\section{COMPARISON OF CO-ADDED SPECTRAL ABUNDANCES AND WEIGHTED-AVERAGE ABUNDANCES}

The test we conduct in this study is to measure abundances on co-added spectra and see if the results we get are consistent with the measured abundances of the input RGB stars. In future applications, the individual star will be too faint to measure abundance, so any biases should be anticipated by using this nearby star sample. In this section, we compared the weightedaverage abundances of each bin with the abundances measured from the co-added spectra in order to test the feasibility of the co-addition method. The spectra we used in this test have a relatively high $\mathrm{S} / \mathrm{N}$, and the final goal of our work is to apply this method to more distant and fainter RGB stars beyond the Milky Way system.

\subsection{Weighted-average Abundances}

In this and subsequent sections, we refer to "weightedaverage" abundances, which are the weighted ensemble averages of the measured abundances for individual stars in a given bin. Correspondingly, the "co-added" abundances are those derived from a measurement on the co-added spectra.

We have tried different weights to combine the individual abundances in bins, then compared weighted-average abundances with the abundances measured from co-added spectra. We found that the same weights that we used in the combining of individual spectrum in co-addition (described in Section 3.4) were the best weights to use if we wanted the two procedures to be consistent and produce the most unbiased results. Taking $[\mathrm{Fe} / \mathrm{H}]$ as an example, we used the elemental mask for Fe, which covers the wavelength regions used to determine $[\mathrm{Fe} / \mathrm{H}]$, resulting in an inverse variance array. The average inverse variance across the entire spectrum, calculated as in Equations (2) and (3), is then used as the weight for that star when combining the individual abundances together to create the weighted-average abundance for that bin. The weights $\omega_{j}$ used for individual abundances in the weighted average are

$$
\begin{gathered}
\sigma_{\text {spec }, j}^{2}=\left(\sum_{i=1}^{\text {mpixel }}\left(\sigma_{\text {pixel }, i j}\right)^{-2} \cdot M_{\text {elemental }, X}\right)^{-1} \\
\omega_{j}(X)=\frac{1 / \sigma_{\text {spec }, j}^{2}}{\sum_{j=1}^{n} 1 / \sigma_{\text {spec }, j}^{2}} .
\end{gathered}
$$

In Equation (2), $\sigma_{\text {pixel, } i j}^{2}$ is the variance of $i$ th pixel of $j$ th spectrum in the bin. mpixel is total number of pixels in a spectrum. $M_{\text {elemental } X}$ is the elemental mask for measurement of $X$, where $X$ could be abundances of $[\mathrm{Fe} / \mathrm{H}],[\mathrm{Mg} / \mathrm{Fe}],[\mathrm{Si} / \mathrm{Fe}]$, $[\mathrm{Ca} / \mathrm{Fe}]$, or $[\mathrm{Ti} / \mathrm{Fe}] . M_{\text {elemental }, X}$ is a binary array in which only the pixels that most sensitive to corresponding element $X$ absorption lines are set to $1 . \sigma_{\text {spec, } j}^{2}$ is the weighted variance for the whole $j$ th spectrum. In Equation $(3), \omega_{j}(X)$ denotes the weight of $X$ for $j$ th star, and there are $n$ stars in that group. Then, the weighted-average abundance is

$$
X_{\mathrm{bin}, \mathrm{wa}}=\sum_{j=1}^{n} \omega_{j}(X) X_{j}=\frac{\sum_{j=1}^{n}\left(X_{j} / \sigma_{\mathrm{spec}, j}^{2}\right)}{\sum_{j=1}^{n} 1 / \sigma_{\mathrm{spec}, j}^{2}} .
$$

We calculated the weighted-average abundances of the four $\alpha$-elements in the same way as $[\mathrm{Fe} / \mathrm{H}]$ for each group. 
As mentioned before, some stars' individual element abundances were unavailable and these stars were not included in the co-added spectra, so we ignored them when determined weighted-average abundances.

\subsection{Errors}

We considered two kind of errors that contribute to the scatter in the abundance distribution: fitting error and systematic error. The fitting error was given by the Levenberg-Marquardt algorithm code. The MPFIT program determined the best-fit synthetic spectrum by minimizing $\chi^{2}$ and gave an estimate of the fitting error based on the depth of the $\chi^{2}$ minimum in parameter space. There are many other sources of error like the inaccuracy of atmospheric parameters, imperfect spectral modeling and imprecise continuum placement. We considered all other uncertainties as systematic error. For individual stars, we used abundance error floors derived by Kirby et al. (2010) as systematic error. ${ }^{12}$ Therefore, the total error for individual stars, $\sigma_{\text {total }, j}$ for the $j$ th star, is calculated as

$$
\sigma_{\text {total }, j}(X)=\sqrt{\left(\sigma_{\text {fit }, j}(X)\right)^{2}+\left(\sigma_{\text {sys }}(X)\right)^{2}},
$$

where $\sigma_{\mathrm{fit}, j}(X)$ is the fit error for abundance $X$ of the $j$ th star, and $\sigma_{\text {sys, }, j}(X)$ is systematic error. The fit error and systematic error for individual stars should be uncorrelated, in which case the total error $\left(\sigma_{\text {total }, j}(X)\right)$ is simply the fit error $\left(\sigma_{\mathrm{fit}, j}(X)\right)$ and systematic error $\left(\sigma_{\mathrm{sys}, j}(X)\right)$ added in quadrature, where $X$ is either $[\mathrm{Fe} / \mathrm{H}]$ or $[\alpha / \mathrm{Fe}]$, and $\alpha$ denotes $\mathrm{Mg}, \mathrm{Si}, \mathrm{Ca}$, or Ti.

For the weighted-average abundance $X_{\text {bin,wa }}$, we estimated the variance for each bin weighted by $\sigma_{\text {total }, j}^{2}(X)$ which are same weights used for weighted-average abundances (see Equations (3) and (4)):

$$
\sigma_{\text {bin }, \text { wa }}^{2}(X)=\omega_{j}^{2} \sigma_{\text {total }, j}^{2}=\frac{\sigma_{\text {total }, j}^{2} / \sigma_{\mathrm{spec}, j}^{4}}{\left(\sum_{j=1}^{n} 1 / \sigma_{\text {spec }, j}^{2}\right)^{2}},
$$

the $\sigma_{\text {bin,wa }}(X)$, then, is the weighted error of weighted mean abundance $X_{\text {bin,wa }}$.

For abundances derived from the co-added spectra, we tried to use the same method in Kirby et al. (2010) to estimate the systematic errors. The distribution of the difference between the measured co-added values and the weighted mean values for same bins, divided by the expected errors, should be well fit by a Gaussian with unit variance, as shown in Equation (7):

$\operatorname{rms}\left(\frac{X_{\text {coadd }}-X_{\text {bin,wa }}}{\sqrt{\left(\sigma_{\text {fit coadd }}(X)\right)^{2}+\left(\sigma_{\text {bin, wa }}(X)\right)^{2}+\left(\sigma_{\text {sys,coadd }}(X)\right)^{2}}}\right)=1$,

where $\sigma_{\text {fit,coadd }}(X)$ is the fitting error of the co-added results, $\sigma_{\text {bin,wa }}(X)$ is weighted mean error calculated from Equation (6), and $\sigma_{\text {sys,coadd }}(X)$ represents the systematic error for the coadded results. These three types of errors are supposed to be independent to each other. In our case, however, the fitting errors $\left(\sigma_{\text {fit,coadd }}(X)\right)$ and weighted mean errors $\left(\sigma_{\text {bin,wa }}(X)\right)$ are already large enough, such that it is impossible to estimate systematic errors for the co-added results from Equation (7). Figure 5 shows the distributions of the difference, divided by

\footnotetext{
12 Table 5 of abundance error floors for five elements in Kirby et al. (2010). Data used in this work source from previous work of Kirby et al.; hence, we expect the systematic errors are same for our measurements.
}

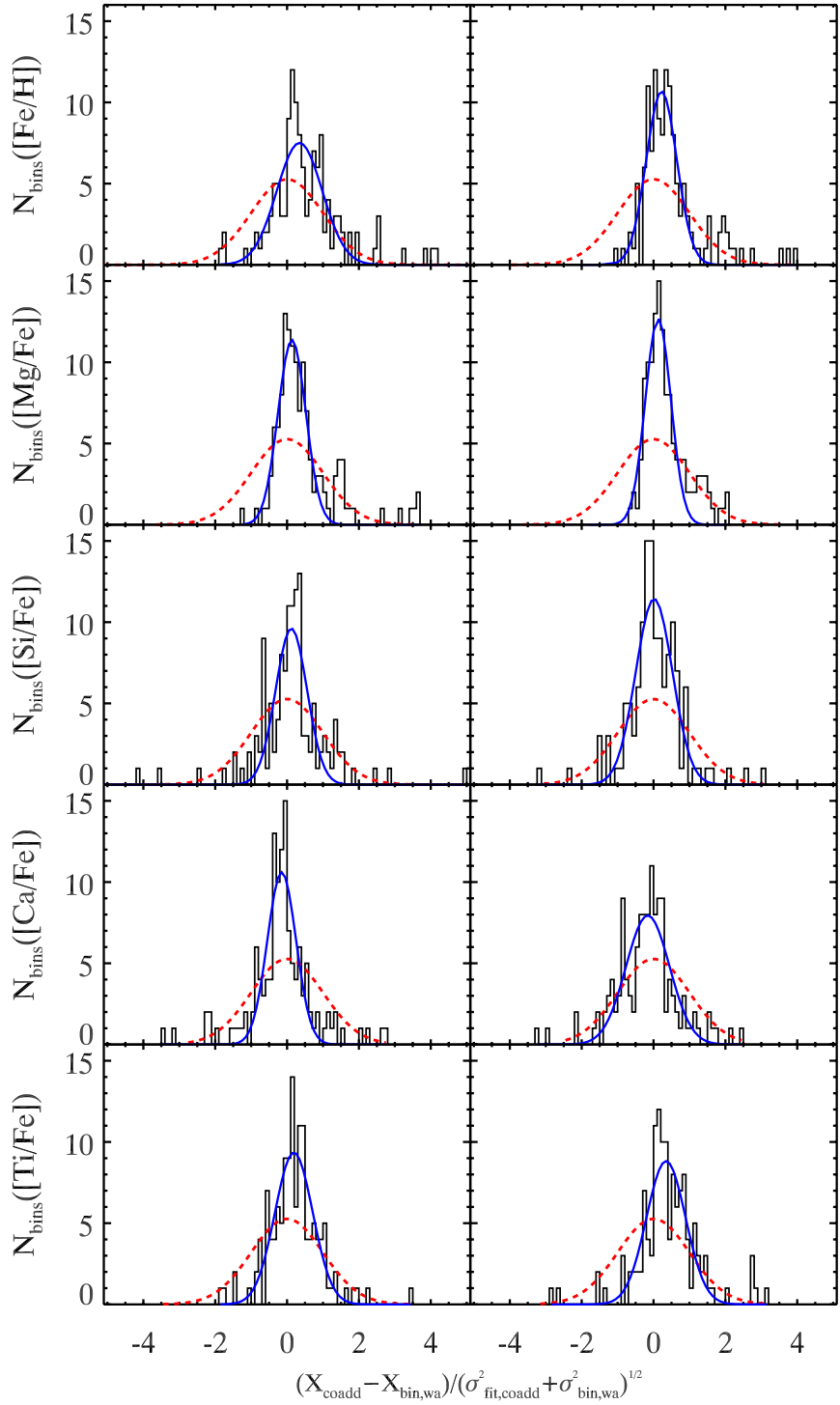

Figure 5. Distribution of the difference between the measurements from co-adds and weighted mean for 132 bins from eight GCs and eight dSphs divided by the errors of difference; systematic errors for co-adds are not included. Distributions in left panels are stars binned by $\left(T_{\text {eff }}\right)_{\text {phot }}$, right panels show stars binned by $[\mathrm{Fe} / \mathrm{H}]_{\text {phot }}$. The solid blue curves are the best-fit Gaussian for distributions. The dashed red curved are unit Gaussian with $\sigma=1$. The areas of the unit Gaussian are normalized to the number of bins.

(A color version of this figure is available in the online journal.)

the expected errors, and not including $\sigma_{\text {sys,coadd }}(X)$, for all $\mathrm{GC}$ and dSph bins. The best-fit Gaussian is narrower than the unit Gaussian in all distributions, indicating that either the differences of the two quantities in the numerator are too small, or the errors in the denominator are too large. However, this does not mean we have overestimated our uncertainties. Since we use the exact same stars for uncertainty estimating, they should contribute both to the weighted-average and to the coadded spectral measurements. It is possible that the random and systematic errors in the mean abundance of each bin cancel out to some degree. These could include errors such as those related to a spread of intrinsic abundances within a bin, a spread caused by spectral noise, and systematic errors resulting from $T_{\text {eff }}$ mismatch. Some of the errors are correlated between the two quantities in the numerator and cancel out when we take the differences. On the other hand, the co-addition enhances 

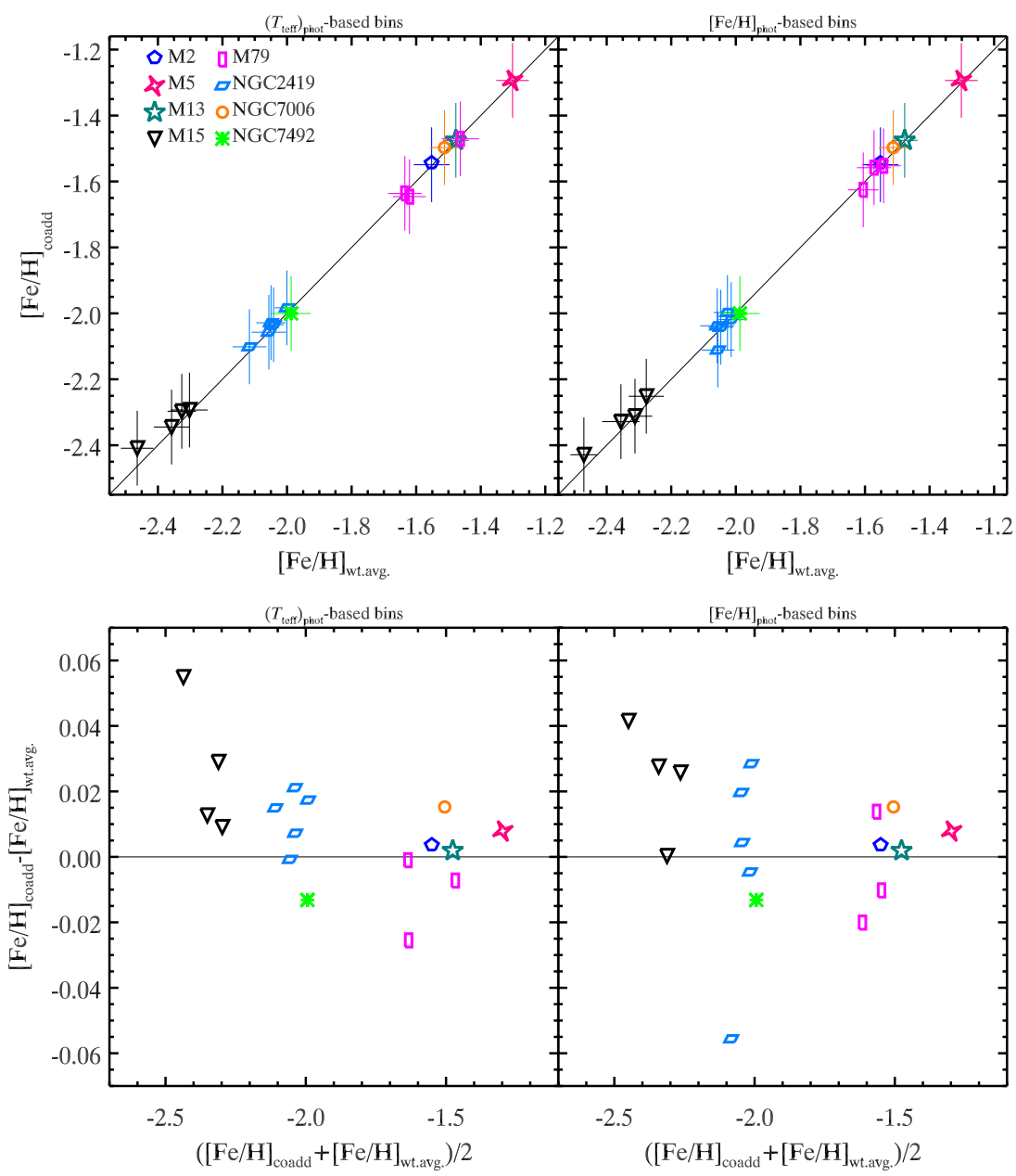

Figure 6. Comparison between weighted-average metallicity and metallicity from co-added spectra for eight GCs in the Milky Way. Top: the value of the vertical axis represents co-added results and horizontal axis is weighted-average results. For weighted-average abundances, we measured every star's abundance and averaged the abundances, weighting by the elemental mask weights that we used in the combining of the individual spectra. We selected stars with log $g \leqslant 1.4$ for all eight GC stars and binned stars by photometric effective temperature (left) and photometric metallicity (right), respectively. Bottom: residuals for co-added and weighted-average metallicity values vs. their average. Each GC has been denoted by an unique symbol and color.

(A color version of this figure is available in the online journal.)

$\mathrm{S} / \mathrm{N}$ of the spectra, then some flavors of systematic errors on the measured abundances, e.g., those resulting the photometric estimate of $T_{\text {eff }}$ being different from the true $T_{\text {eff }}$, may indeed average down in the case of abundance determination from the co-added spectrum. So we expect the systematic errors for coadded abundances should be smaller than the systematic error floors of individual stars. If the errors are correlated at some level, then there must be a non-negligible negative covariance term in the denominator. Both effects could be going on in our case. Here, we make a simplification for the systematic errors of the co-added results that we used the abundance error floors of individual stars for the co-added uncertainties and calculated them as

$$
\sigma_{\text {total }, \text { coadd }}(X)=\sqrt{\left(\sigma_{\text {fit, coadd }}(X)\right)^{2}+\left(\sigma_{\text {sys }}(X)\right)^{2}}
$$

where $\sigma_{\text {fit,coadd }}(X)$ is the fit error of co-added abundance from Equation (7). $\sigma_{\mathrm{sys}}(X)$ is the systematic error from Equation (5). $\sigma_{\text {total,coadd }}(X)$ is the total error used for co-added abundances. As Figure 5 shows, our error estimates are conservative. The true errors, accounting for covariance, must be slightly smaller. For the RGB stars of M31 satellites, there will be non-negligible random errors that result from the analysis of low-S/N spectra. Random errors often have a Gaussian normal distribution and contribute to the total errors in the measurements. We will discuss the effects of random errors to the error budget in future work of M31.

\subsection{Comparison}

We expect that the abundances measured on the co-added spectra should match the weighted-average abundances of the stars that were used to produce the co-add. By using high-S/N medium-resolution spectra of nearby RGB stars, we can know both the input and output abundances and make a robust comparison. The co-added abundances for eight GCs and eight dSphs are derived from co-added spectra as described in Section 3.4, and the weighted-average abundances are calculated based on individual abundances from Section 3.2 and combined as described in Section 4.1.

For eight GCs, the test is cleaner because we can reasonably assume no age, metallicity, or $\alpha$ abundance spread. Figure 6 shows the comparisons between weighted-average metallicity and co-added spectra metallicity. Stars used here are constrained by $\log g(\leqslant 1.4)$ and binned by photometric effective temperature 

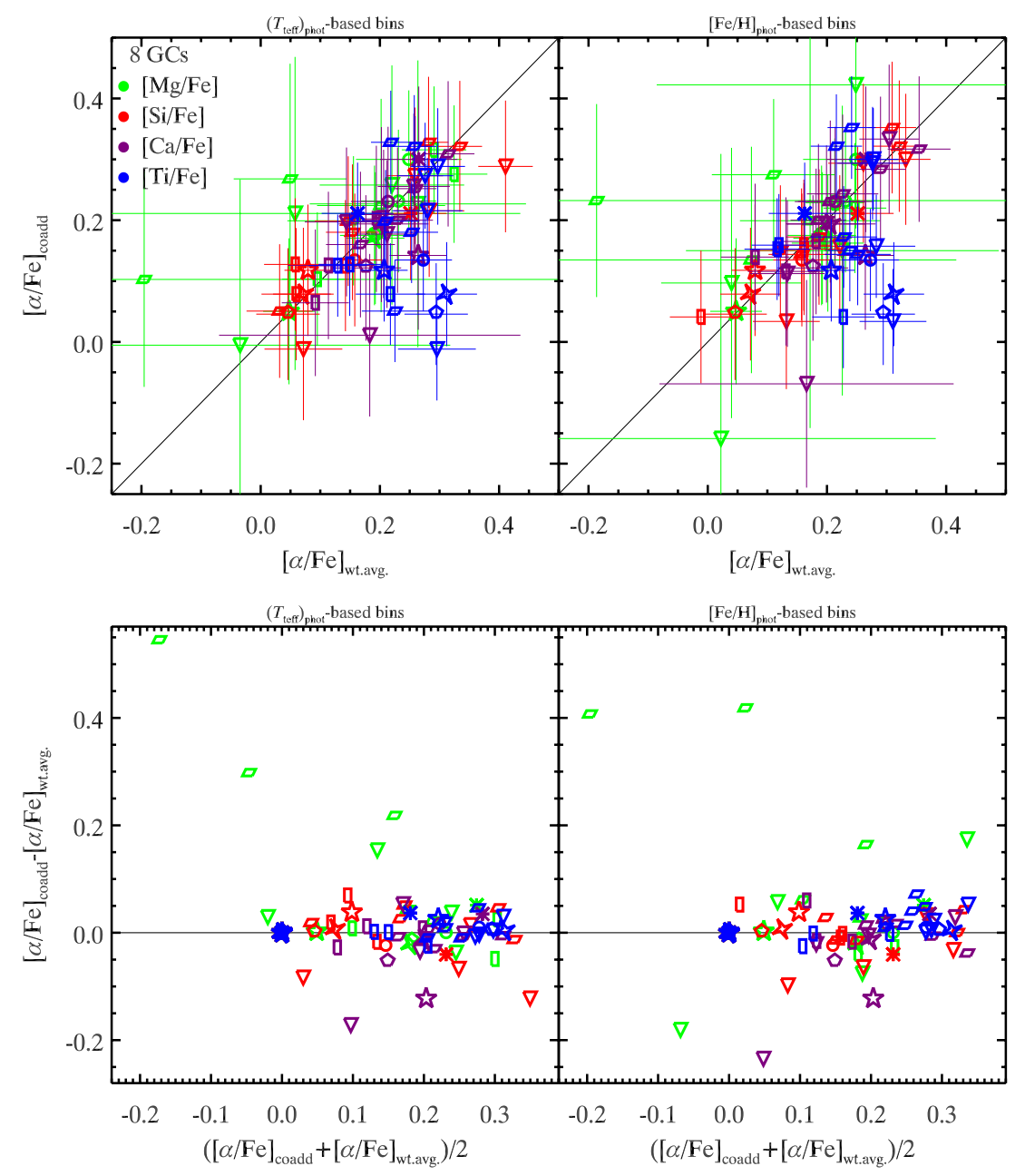

Figure 7. Same as Figure 6 except for $[\alpha / \mathrm{Fe}]$ instead of $[\mathrm{Fe} / \mathrm{H}]$. The different symbols denote the different GCs as defined in Figure 6 but with different colors for the different $\alpha$-elements. $[\mathrm{Mg} / \mathrm{Fe}]$ in green, $[\mathrm{Si} / \mathrm{Fe}]$ in red, $[\mathrm{Ca} / \mathrm{Fe}]$ in purple, and $[\mathrm{Ti} / \mathrm{Fe}]$ in blue.

(A color version of this figure is available in the online journal.)

(left panels) and photometric metallicity (right panels). GCs are monometallic, so the spreads in the photometric metallicity of GCs are expected to arise from measurement uncertainty. Despite the limited number of stars in the GCs, the two results demonstrate a high level of agreement for these bins (top panels). The differences between the co-added metallicities and weighted-average metallicities are less than 0.06 dex for both binned scenarios as shown in the bottom panels. In Figure 7 , the comparisons of $\alpha$-elements on a bin basis also match well, except $[\mathrm{Mg} / \mathrm{Fe}]$, for which the co-added results present relatively larger values compared to the weightedaverage results, especially for NGC 2419 as shown in the two bottom panels of residuals.

For eight dSphs, Figure 8 shows metallicity comparisons between weighted-mean and co-added spectra measurements. The plots on the left show bins of stars grouped by photometric $T_{\text {eff }}$ and the plots on the right show bins grouped by $[\mathrm{Fe} / \mathrm{H}]_{\text {phot }}$. Each point represents one bin of stars. The two bottom plots present the difference of the two results versus their straight averages. The two results show a good match over $-1.7<$ $[\mathrm{Fe} / \mathrm{H}]<-0.7$, for which the difference less than 0.05 dex. For $[\mathrm{Fe} / \mathrm{H}] \lesssim-1.5$ dex, the abundances from co-added spectra show a slight bias to be more metal-rich for both binning approaches, and stars binned by $\left(T_{\text {eff }}\right)_{\text {phot }}$ show slightly less scatter. We use a high-order polynomial fit to compare the bias trends of the residuals for the two binning scenarios. There are total 115 bins for each scenario. For stars binned by photometric effective temperature, around $96 \%$ of bins have residuals less than $0.1 \mathrm{dex}$, and for photometric metallicity, around $95 \%$ of bins have residuals less than 0.1 dex.

Figure 9 shows the comparison of four $\alpha$-elements for eight dSphs. Even after limiting our sample to $\log g \leqslant 1.4$, i.e., the brighter stars were chosen, some stars still have unmeasurable elemental abundances and are not included in the bins. The plots in Figure 10 show the difference of two measurements as in the bottom panels in Figure 8. The scatter here is larger than for $[\mathrm{Fe} / \mathrm{H}]$, especially for $[\mathrm{Mg} / \mathrm{Fe}]$, for which the co-added spectra consistently scatter to higher values. The same trend seen in the GC stars is shown in Figure 7.

There are many factors that can cause a discrepancy between these two mean abundances, such as $\mathrm{S} / \mathrm{N}, T_{\text {eff }}$, and $\log g$, all of which may be interrelated. Since brighter RGB stars (with high $\mathrm{S} / \mathrm{N}$ ) often have lower $\log g$ and lower $T_{\text {eff }}$, it is difficult to untangle which of these stellar parameters affecting the abundance measurements most. For dSphs, there is a spread of ages and metallicities, which removes some of the degeneracy between $\mathrm{S} / \mathrm{N}$ and $T_{\text {eff }}$ and $\log g$, but our current data set still cannot sort this out. Figures 11 and 12 show the impact of 

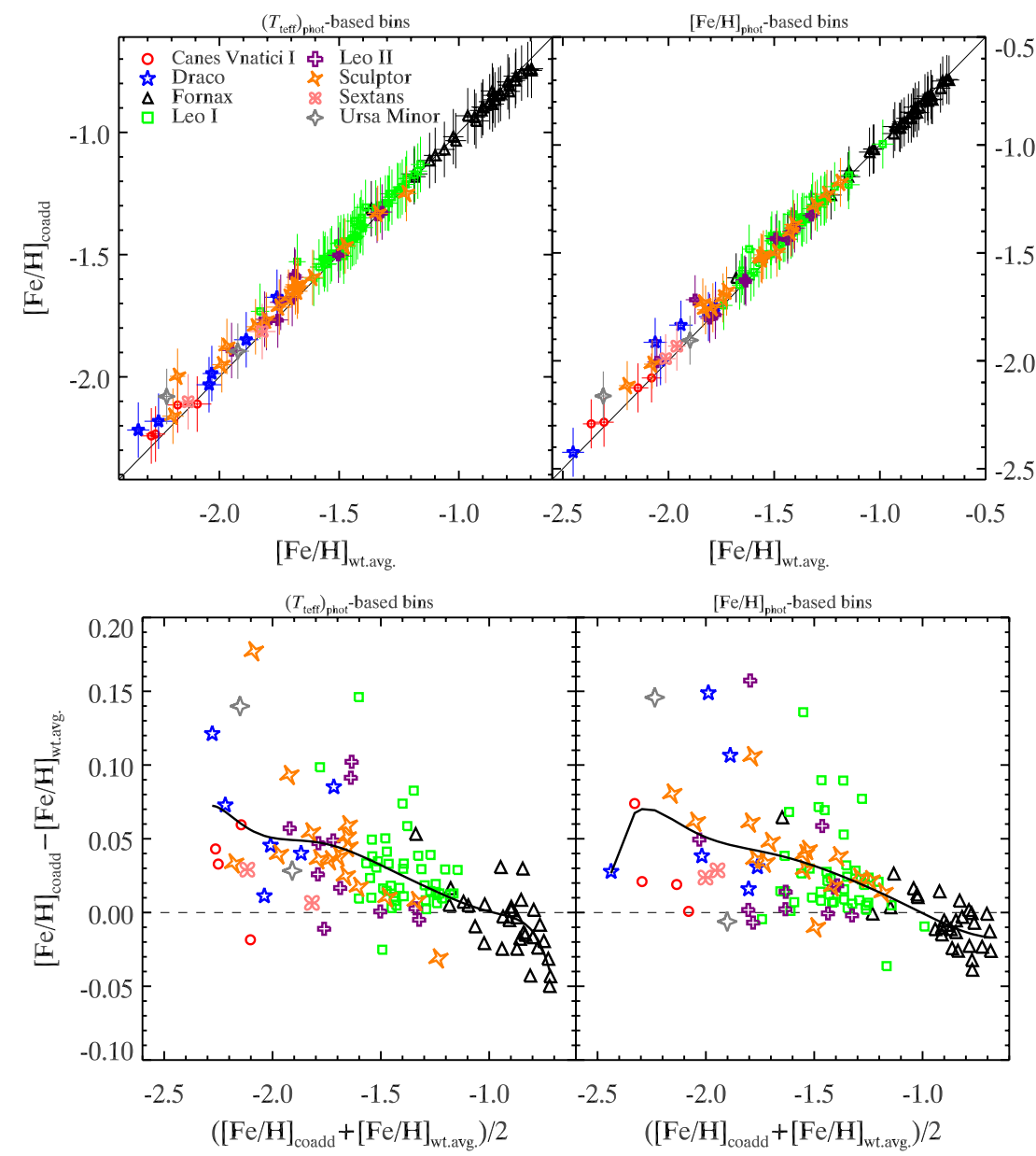

Figure 8. Comparison between weighted-average metallicity and metallicity from co-added spectra for eight dSphs in the Milky Way. Unique symbols with different colors have been used to denote the eight $\mathrm{dSphs}$ in the plots. Top: the value of the vertical axis represents co-added results and horizontal axis is weighted-average results. For weighted-average abundances, we measured every star's abundance and averaged the abundances, weighting by the elemental mask weights which are used in the combining of individual spectrum in co-adding spectrum. We selected stars with $\log g \leqslant 1.4$ for all eight dSph stars and binned stars by photometric effective temperature (left) and photometric metallicity (right), respectively. Bottom: residuals between $[\mathrm{Fe} / \mathrm{H}]_{\text {coadd }}$ and $[\mathrm{Fe} / \mathrm{H}]_{\text {wt.avg. }}$ vs. the average of $[\mathrm{Fe} / \mathrm{H}]_{\text {coadd }}$ and $[\mathrm{Fe} / \mathrm{H}]_{\mathrm{wt} \text {.avg. }}$. The curves in black are derived by polynomial fitting the residuals.

(A color version of this figure is available in the online journal.)

$\mathrm{S} / \mathrm{N}$ values for GCs and dSphs, respectively. In the left panels the stars are binned by $\left(T_{\text {eff }}\right)_{\text {phot }}$ and the stars are binned by $[\mathrm{Fe} / \mathrm{H}]_{\text {phot }}$ in the right panels. The dashed lines show the standard deviations for the differences. Here we calculate the $\mathrm{S} / \mathrm{N}$ of the co-added spectrum weighted by the inverse variance:

$$
\left\langle\mathrm{S} / \mathrm{N}_{\mathrm{coadd}}\right\rangle=\frac{\sum_{i=1}^{n}\left(1 / \sigma_{\mathrm{pixel}, i}\right) \cdot\left(1 / \sigma_{\mathrm{pixel}, i}^{2}\right)}{\sum_{i=1}^{n} 1 / \sigma_{\mathrm{pixel}, i}^{2}},
$$

where $\mathrm{S} / \mathrm{N}_{\text {coadd }}$ is the signal-to-noise ratio for co-added spectrum and $\sigma_{\text {pixel }, i}$ is the error of the $i$ th pixel of co-added spectrum. $\mathrm{GCs}$ have much higher $\mathrm{S} / \mathrm{N}$ for their co-added spectra compared to dSphs. For dSph stars binned by $\left(T_{\text {eff }}\right)_{\text {phot }}$, the discrepancies are slightly pronounced with $\mathrm{S} / \mathrm{N}$ decreasing, and seem to be more concentrated compared with the stars binned by $[\mathrm{Fe} / \mathrm{H}]_{\text {phot }}$ in Figure 12. In general, however, the abundances are in good agreement for both binning methods, within 0.1 dex for GCs and within 0.2 dex for dSphs, with $[\mathrm{Mg} / \mathrm{Fe}]$ having the most outliers.

Figures 13 and 14 consider the impact of $\left\langle T_{\text {eff }}\right\rangle$ for the abundance discrepancies. For each bin, We combined the individual $T_{\text {eff }}$ weighted by the total error of the star to calculate the mean $\left\langle T_{\text {eff }}\right\rangle$. The left panels show stars grouped by $\left(T_{\text {eff }}\right)_{\text {phot }}$ while the right panels show stars binned by $[\mathrm{Fe} / \mathrm{H}]_{\text {phot }}$. The dashed lines represent one standard deviation. There is no obvious trend shown in Figure 13 for GCs, but for dSphs, there appears a slight bias in $[\mathrm{Fe} / \mathrm{H}]$, where $[\mathrm{Fe} / \mathrm{H}]_{\text {coadd }}$ is lower at lower $\left\langle T_{\text {eff }}\right\rangle$. Also, the differences in $[\mathrm{Mg} / \mathrm{Fe}]$ are markedly higher when $4400 \mathrm{~K} \leqslant\left\langle T_{\text {eff }}\right\rangle \leqslant 4600 \mathrm{~K}$ for both binning approaches.

Figures 15 and 16 consider the impact of $\langle\log g\rangle$ for the abundance discrepancies. The photometric $\langle\log g\rangle$ for each bin is derived by combining individual values weighted with total error of star. Like $\left\langle T_{\text {eff }}\right\rangle$, the differences in $[\mathrm{Mg} / \mathrm{Fe}]$ are tied to $\langle\log g\rangle$, particularly for stars binned by $T_{\text {eff }}$ with larger scatter for $\langle\log g\rangle>0.9$. The discrepancies in metallicities and $\alpha$-elements for GCs seem to be not very sensitive to $\left\langle\mathrm{S} / \mathrm{N}_{\text {coadd }}\right\rangle,\left\langle T_{\text {eff }}\right\rangle$, or $\langle\log g\rangle$, and we suspect the limited number of bins (only 17 bins for GCs) is one reason.

\subsubsection{Photometric Metallicity}

In order to make a robust comparison, we also compared metallicity derived from co-added spectra $\left([\mathrm{Fe} / \mathrm{H}]_{\text {coadd }}\right)$ to the weighted average of photometric metallicity $\left([\mathrm{Fe} / \mathrm{H}]_{\text {phot }}\right)$ as shown in Figure 17. We combined the photometric metallicities of member stars in each bin weighted by their photometric 


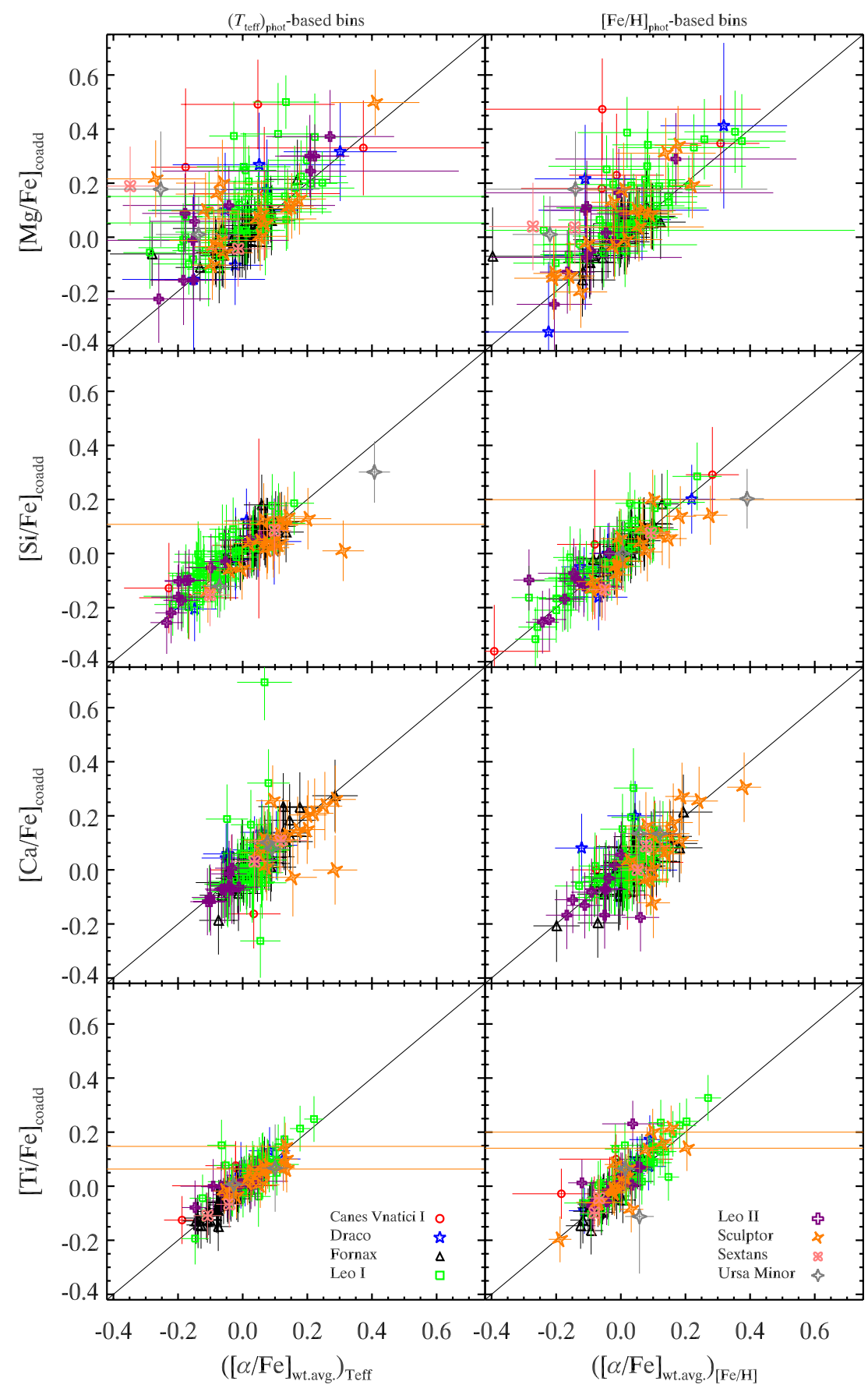

Figure 9. Comparison for $\alpha$-element abundances between weighted-average abundances and co-added spectral abundances for four $\alpha$-elements, $\mathrm{Mg}, \mathrm{Si}, \mathrm{Ca}$, and $\mathrm{Ti}$ (top to bottom). Stars used for these plots are from the eight dSph galaxies. Points in the left panels are stars binned by $T_{\text {eff }}$ and right-hand panels are binned by $[\mathrm{Fe} / \mathrm{H}]_{\text {phot }}$. The symbols are same as in Figure 8.

(A color version of this figure is available in the online journal.)

errors. In the top panels of Figure 17, the photometric metallicities of weighted-average bins present scatter for M15 and NGC 2419, but this is still acceptable given the uncertainty in photometric measurements. NGC 7006 shows the best agreement for both binning methods. For dSphs, as shown in the bottom panels of Figure 17, we roughly get similar results as in Lianou et al. (2011), in which mean the photometric metallicity estimate only has limited reliability. There is a significant scatter in the dSphs, except for Fornax which seems to show a better agreement and one possible reason is the choice of photometric filters. In our data set, the photometry for Fornax is in the $B$ and $R$ bands. Almost all of the other galaxies have photometry in $V / I$ or $M / T_{2}$. The $B$ band is more metallicity sensitive than the $V$ or $M$ bands. Lianou et al. (2011) only considered $V / I$ photometry. Therefore, it is possible a large amount of the uncertainty in $[\mathrm{Fe} / \mathrm{H}]_{\text {phot }}$ comes from a poor choice of filters. We will discuss the impact of our age assumption in Section 4.4.4.

\subsubsection{Degradation of Spectra}

The aim for developing our co-added spectral technique is to use it for abundance measurements of medium-resolution 


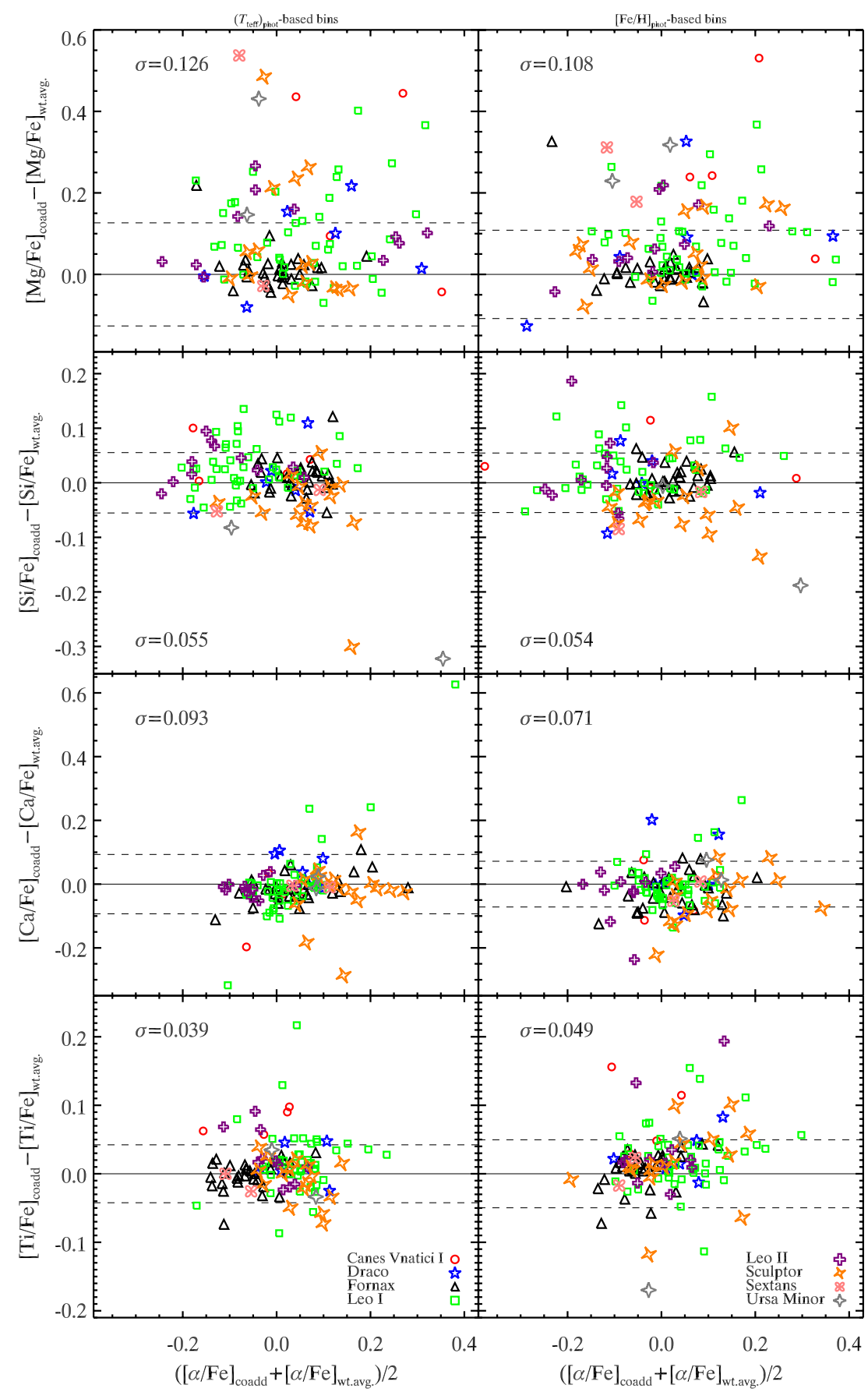

Figure 10. Comparison for $\alpha$-element abundances. The difference between the weighted-average abundance and co-added spectral abundance for four $\alpha$-elements, $\mathrm{Mg}, \mathrm{Si}, \mathrm{Ca}$, and $\mathrm{Ti}$ (top to bottom) vs. the mean abundance. This is the corresponding difference plot for Figure 9. As before, points in the left panels are stars binned by $T_{\text {eff }}$ and right-hand panels are binned by $[\mathrm{Fe} / \mathrm{H}]_{\text {phot }}$. We also calculate the standard deviation for each residual distribution draw as the dashed lines. The symbols are same as in Figure 8.

(A color version of this figure is available in the online journal.)

spectra of RGB stars in M31's dwarf satellite galaxies. The S/N of these stars, however, are far lower (with a typical $\mathrm{S} / \mathrm{N}$ of $<$ $10 \mathrm{pixel}^{-1}$ ) than that for the stars in this test. To complete this test, we reran our codes with degraded spectra and compared these measurements with the weighted-average results derived in Section 4.1. We have randomly chosen $24 \mathrm{RGB}$ stars of NGC 185 and calculated the weighted-average $\mathrm{S} / \mathrm{N}$ of these 24 spectra with Equation (9). We reference arm_addnoise written by Marble (2004) to degrade the high-S/N spectra. We compare the errors $\left(\sigma_{\mathrm{MW}}\right)$ of individual reduced high-S/N spectra with the rebinned errors $\left(\sigma_{\mathrm{M} 31}\right)$ from the weighted-average $\mathrm{S} / \mathrm{N}$ of 24 M31 spectra, to determine the pixels whose $S / N_{M W}$ are higher than $\mathrm{S} / \mathrm{N}_{\mathrm{M} 31}$ to degrade. We degrade the high-S/N spectrum by adding new noise to the spectrum as below:

$$
\begin{gathered}
f_{\text {new }}=f_{\text {old }}+n_{\text {Gaussian }} \cdot\left(\sqrt{\left(\frac{\mathrm{S} / \mathrm{N}_{\mathrm{M} 31}}{\mathrm{~S} / \mathrm{N}_{\mathrm{MW}}}\right)^{-2}-1} \cdot \sigma_{\mathrm{MW}}\right) \\
\sigma_{\text {new }}=\sigma_{\mathrm{MW}} \cdot \frac{\mathrm{S} / \mathrm{N}_{\mathrm{MW}}}{\mathrm{S} / \mathrm{N}_{\mathrm{M} 31}},
\end{gathered}
$$




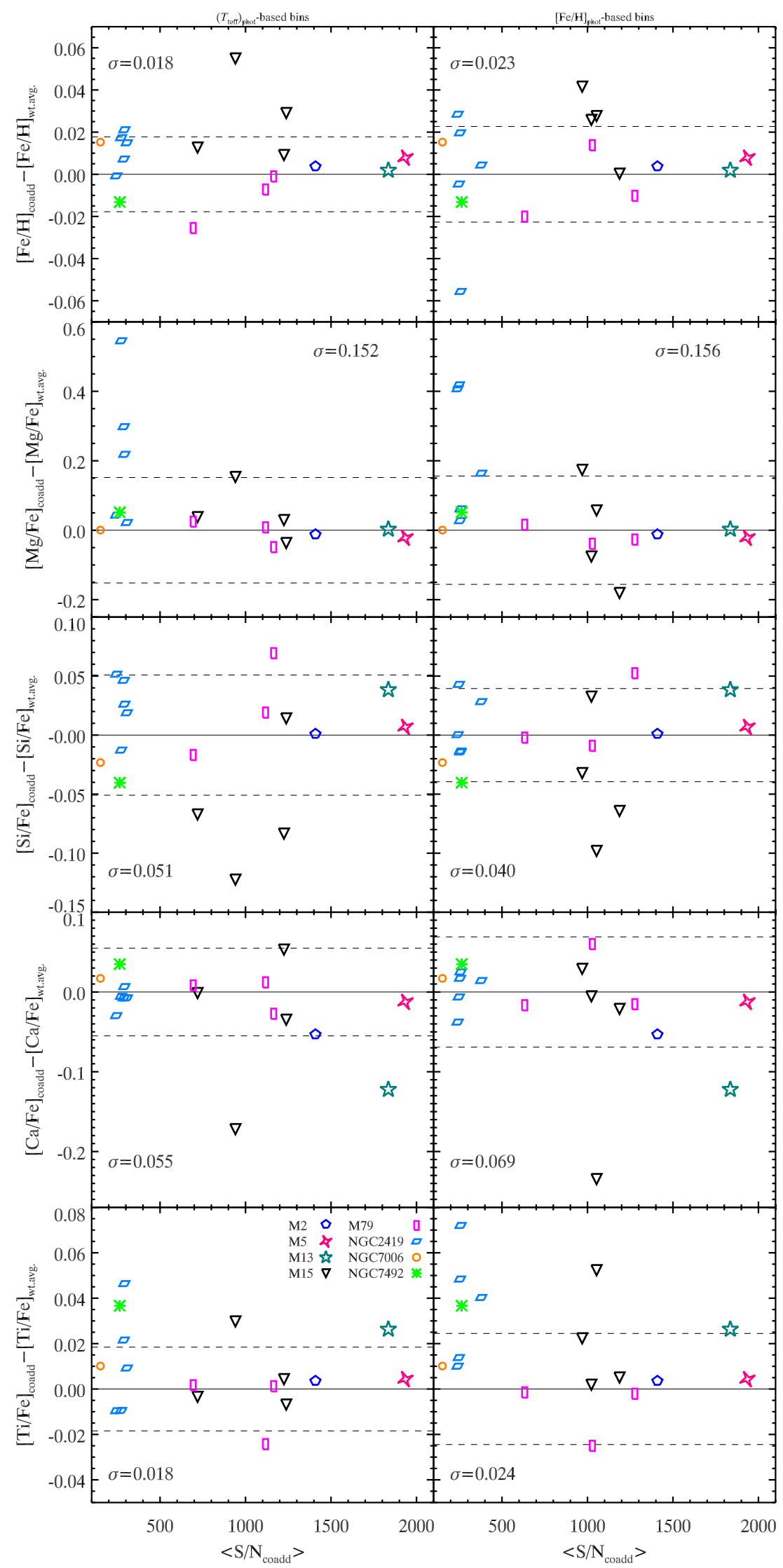

Figure 11. Difference between abundance measurements from co-added spectra vs. the weighted average of individual stellar abundances plotted vs. the $\mathrm{S} / \mathrm{N}$ of co-added spectra of each bin for the eight GCs. The left panels use bins based on photometric estimates of stellar effective temperature $\left(T_{\text {eff }}\right)_{\text {phot }}$, while the right panel used bins based on photometric estimates of metallicity $[\mathrm{Fe} / \mathrm{H}]_{\text {phot }}$. The dashed lines mark the range, $\pm \sigma$, in each panel. The symbols are the same as in Figure 6 . The abundance differences do not show a strong trend with $\mathrm{S} / \mathrm{N}$.

(A color version of this figure is available in the online journal.) 


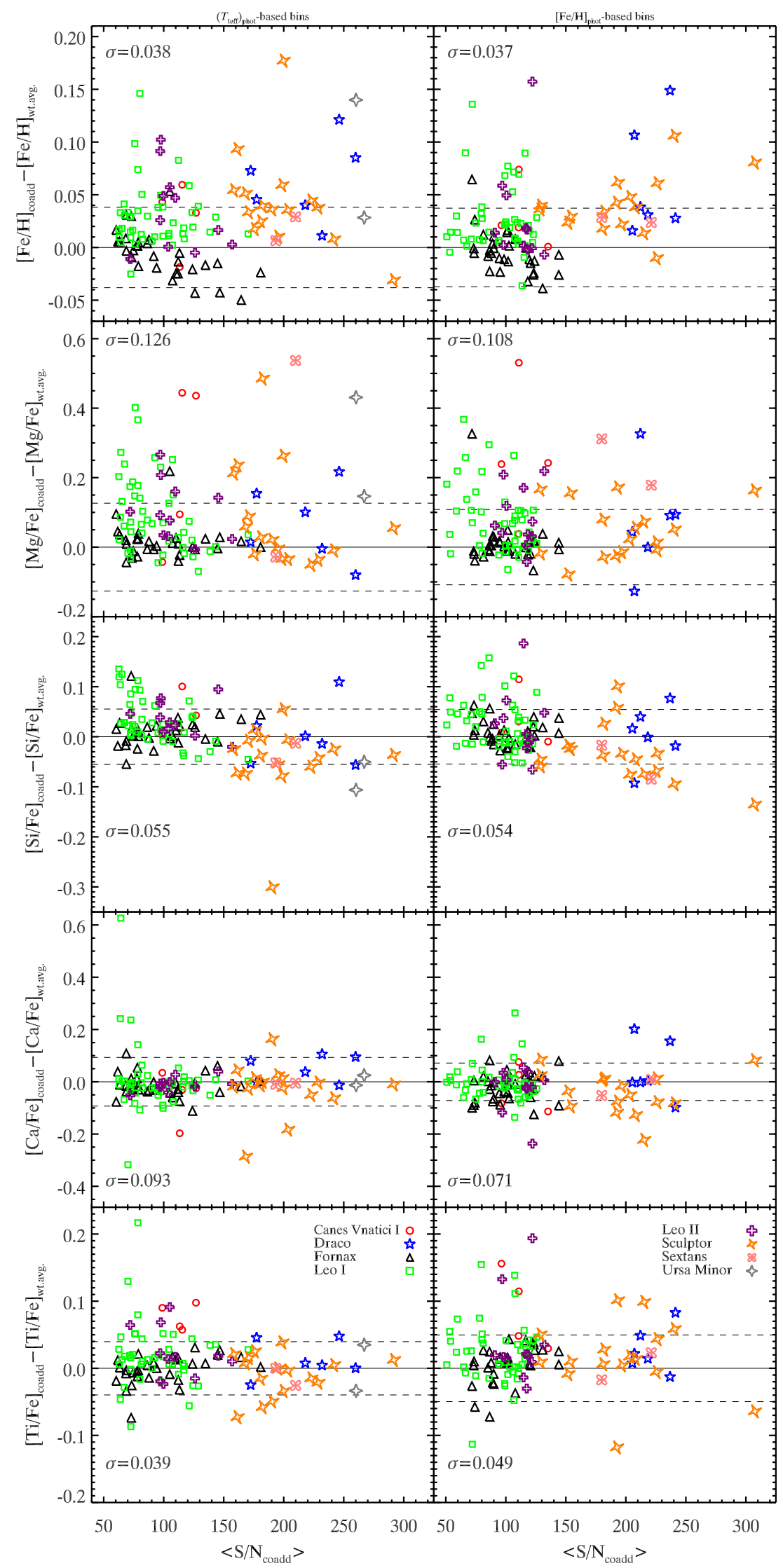

Figure 12. Difference between abundance measurements from co-added spectra vs. the weighted average of individual stellar abundances plotted vs. the $\mathrm{S} / \mathrm{N}$ of co-added spectra of each bin for the eight dSphs. The left panels use bins based on photometric estimates of stellar effective temperature $\left(T_{\text {eff }}\right)_{\text {phot }}$, while the right panel used bins based on photometric estimates of metallicity $[\mathrm{Fe} / \mathrm{H}]_{\text {phot }}$. The dashed lines mark the range, $\pm \sigma$, in each panel. The symbols are the same as in Figure 8 . The abundance differences do not show a strong trend with $\mathrm{S} / \mathrm{N}$.

(A color version of this figure is available in the online journal.) 


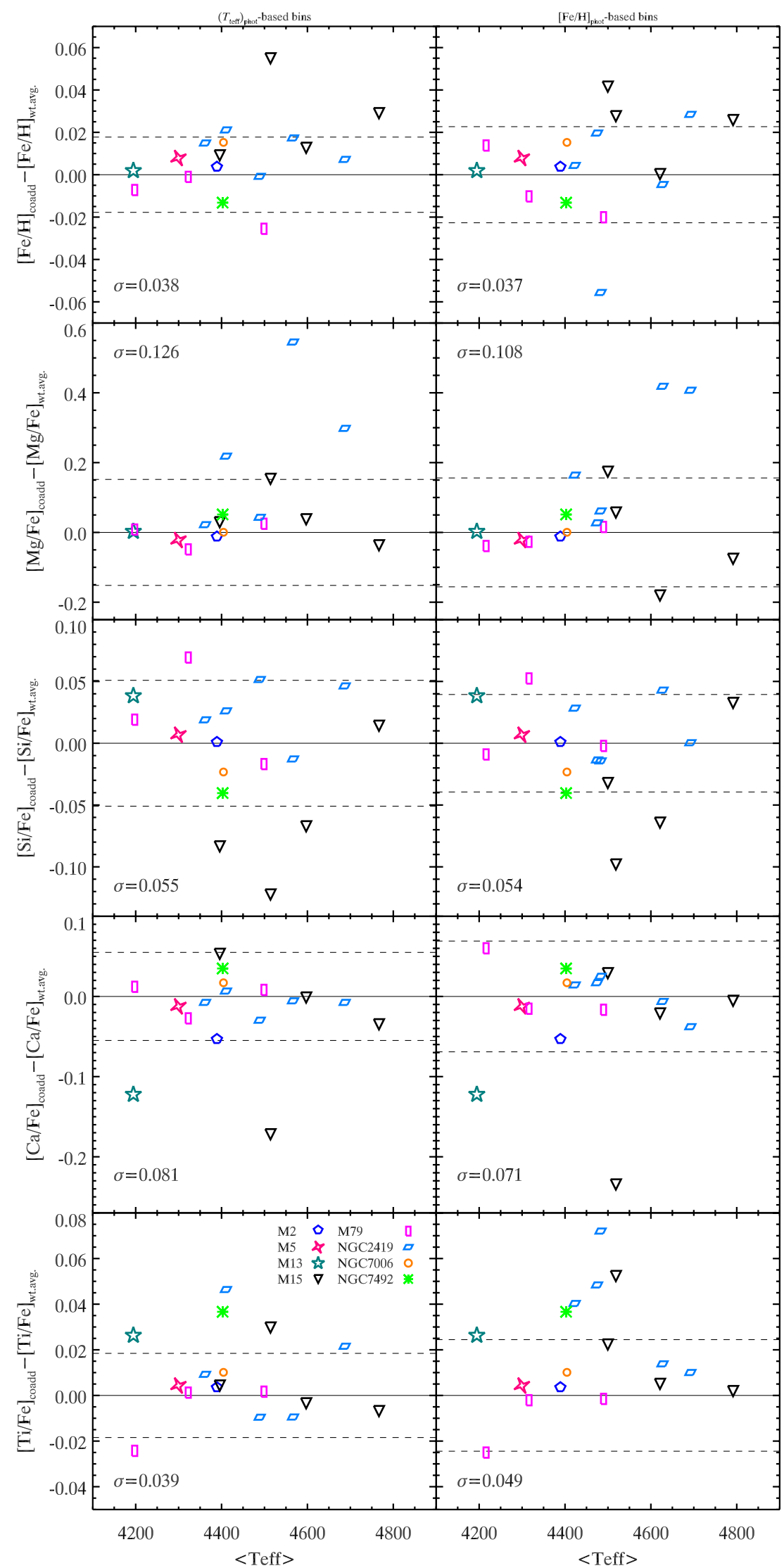

Figure 13. Same as Figure 11 except the abundance differences are plotted vs. mean of stellar effective temperature for each bin. The symbols are the same as in Figure 6.

(A color version of this figure is available in the online journal.) 


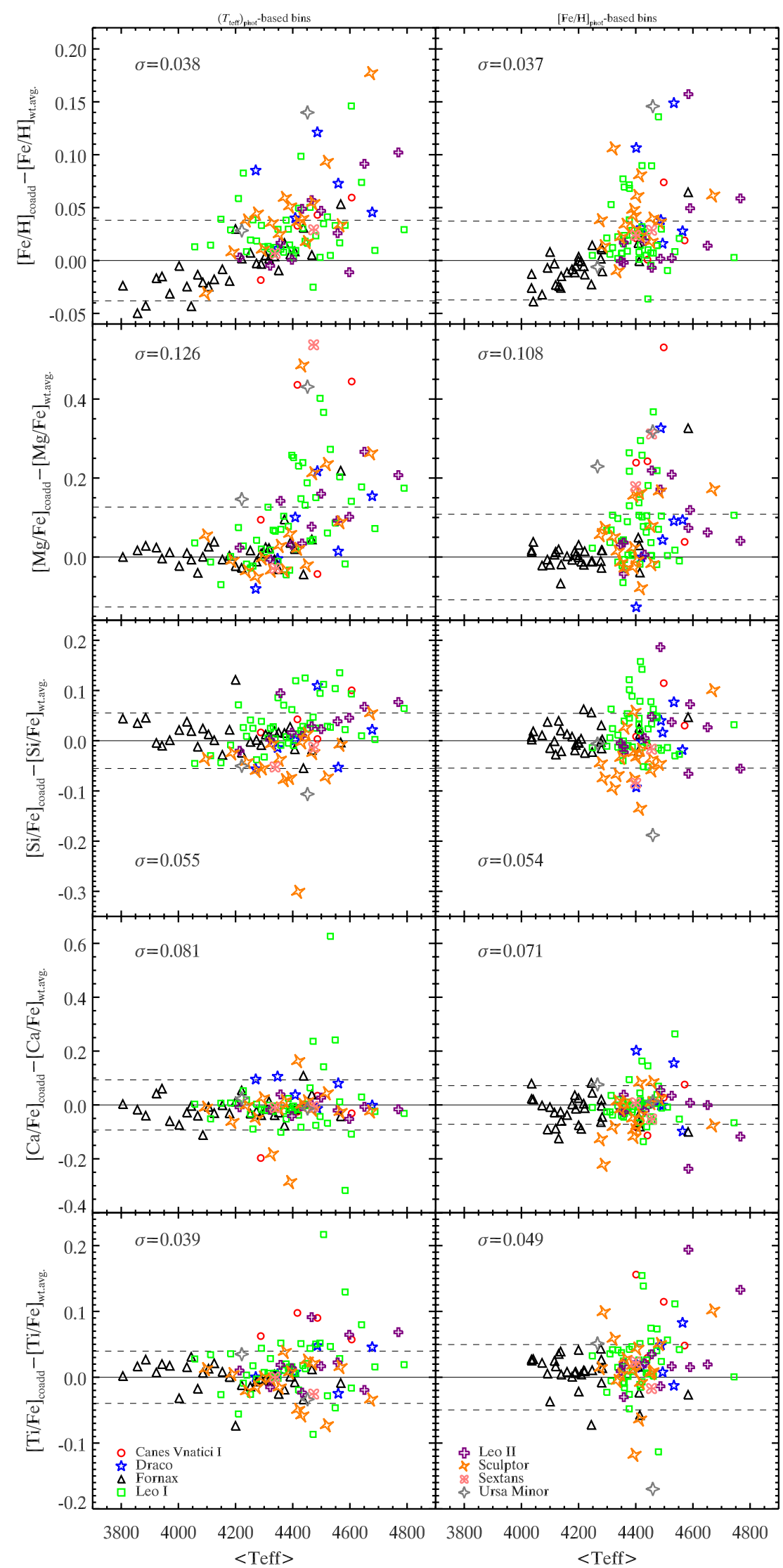

Figure 14. Same as Figure 12 except the abundance differences are plotted vs. mean of stellar effective temperature of each bin. The differences tend to be larger and biased toward positive values at higher $T_{\text {eff }}$ values especially for $[\mathrm{Fe} / \mathrm{H}]$ and $[\mathrm{Mg} / \mathrm{Fe}]$. The symbols are the same as in Figure 8 .

(A color version of this figure is available in the online journal.) 


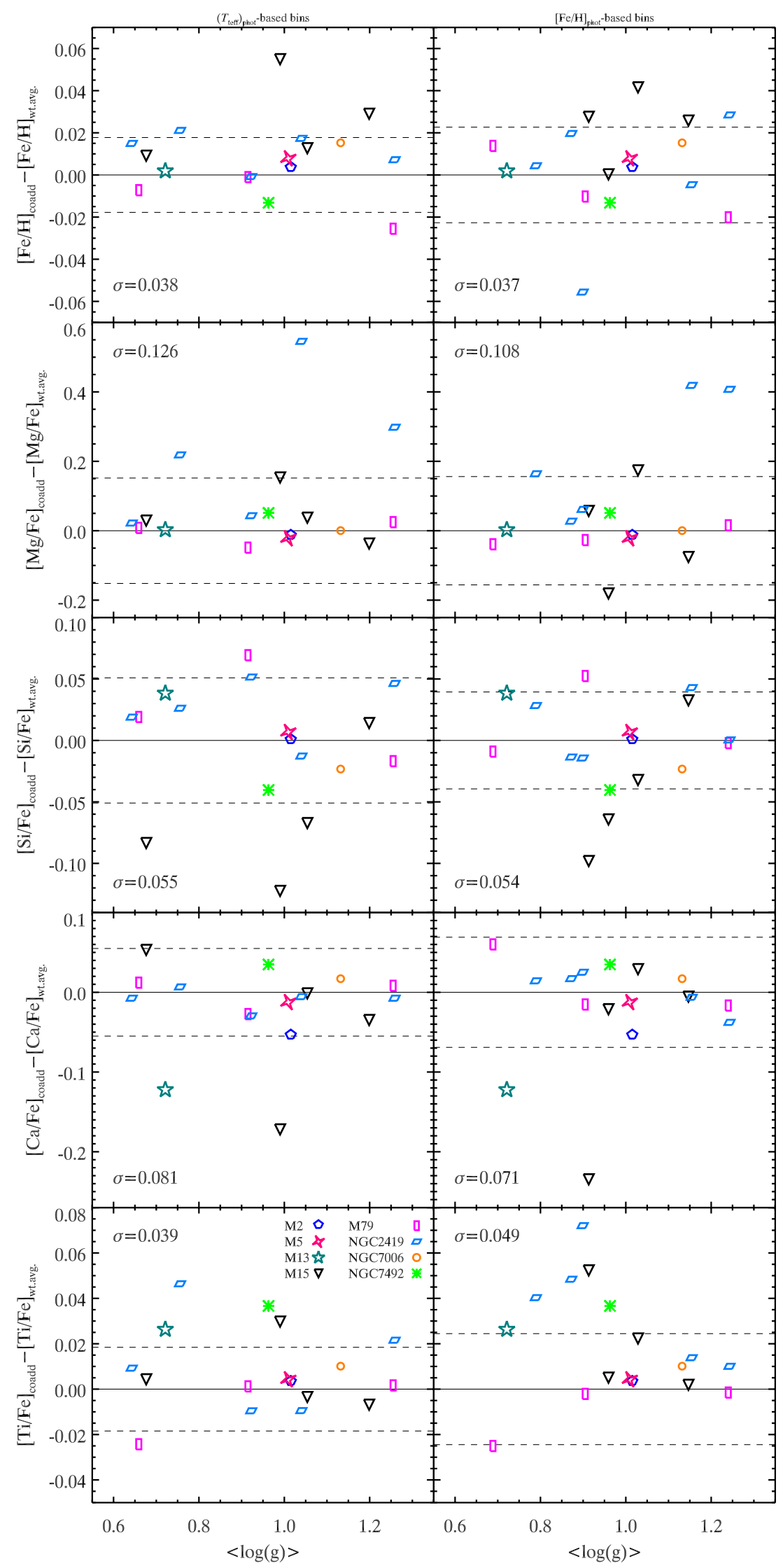

Figure 15. Same as Figure 11 except the abundance differences are plotted vs. mean of stellar surface gravity for each bin. The symbols are same as in Figure 6. (A color version of this figure is available in the online journal.) 


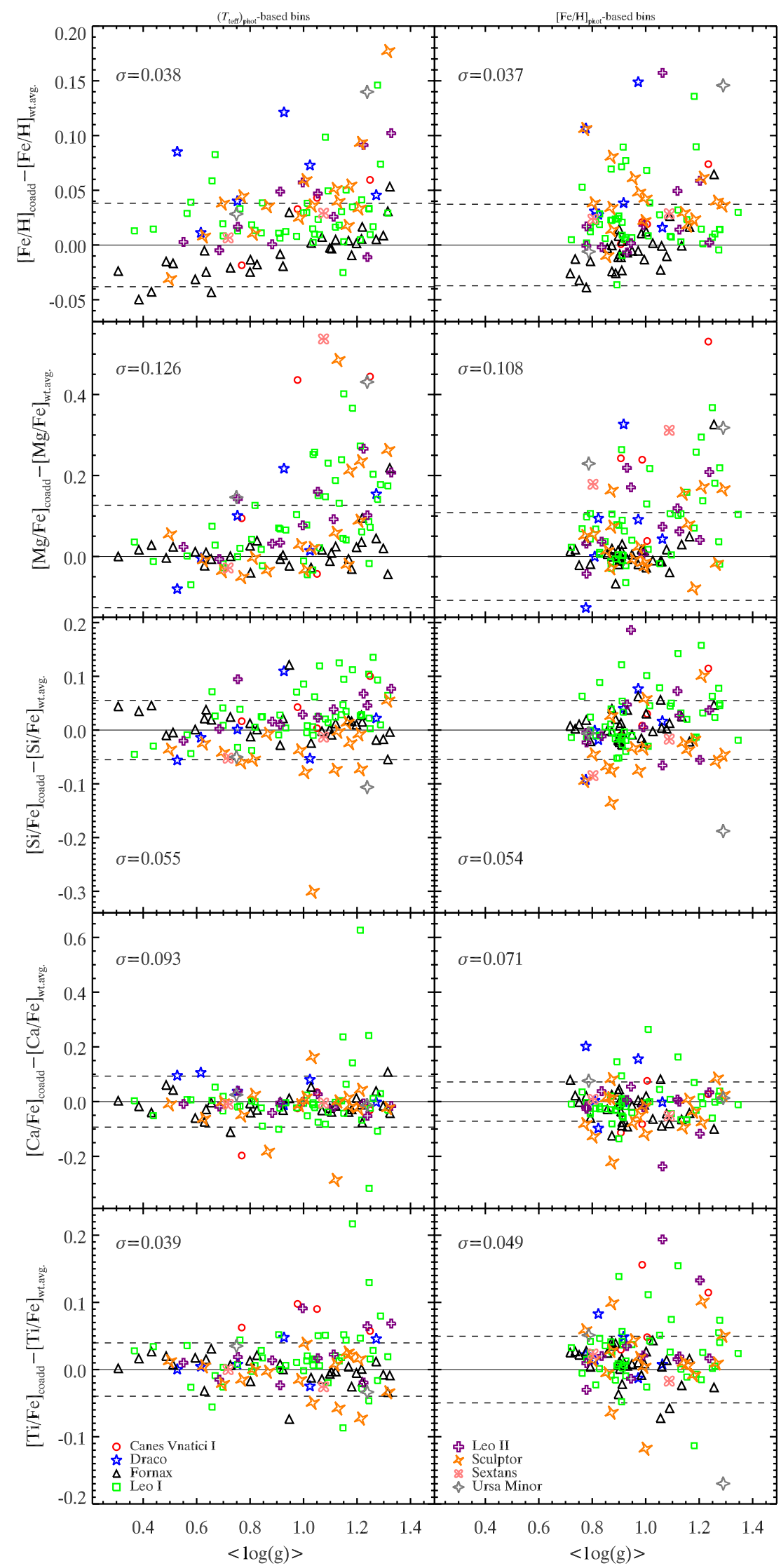

Figure 16. Same as Figure 12 except the abundance differences are plotted vs. mean of stellar surface gravity for each bin. The differences tend to be larger and biased toward positive values at higher $\log g$ values especially for $[\mathrm{Fe} / \mathrm{H}]$ and $[\mathrm{Mg} / \mathrm{Fe}]$. The symbols are same as in Figure 8 .

(A color version of this figure is available in the online journal.) 

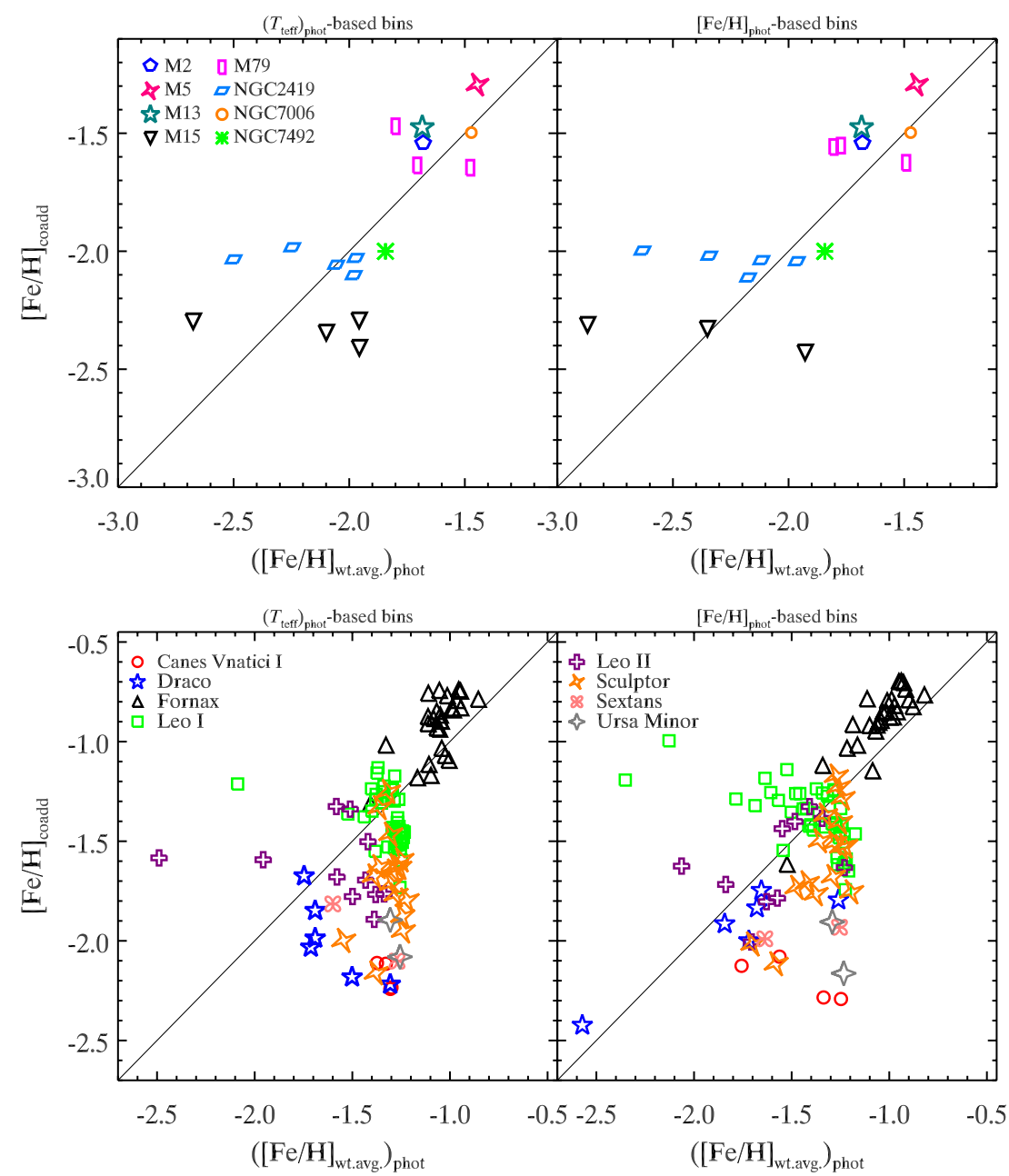

Figure 17. Comparison between weighted-average photometric metallicity and metallicity from co-added spectra for eight GCs (top) and eight dSphs (bottom) in the Milky Way. The value of the vertical axis represents co-added results and horizontal axis is weighted-average results. For weighted-average abundances, we measured every star's metallicity by isochrone fitting with Yonsei-Yale theoretical isochrones. We assumed an age of $14 \mathrm{Gyr}$ for all $\mathrm{RGB}$ stars and $[\alpha / \mathrm{Fe}]=+0.3$. Then we averaged photometric metallicity for each bin weighting by photometric errors. Here all stars satisfy $\log g \leqslant 1.4$. Stars are binned by photometric effective temperature (left) and photometric metallicity (right), respectively. The symbols are same as in Figure 6 for GCs and Figure 8 for dSphs. One bin of M15 has been ignored in the top-right panel whose weighted-average photometric metallicity is less than -3.0 .

(A color version of this figure is available in the online journal.)

where $f_{\text {old }}$ and $f_{\text {new }}$ are the fluxes of high-S/N spectrum before and after added noise, respectively. $n_{\text {Gaussian }}$ is Gaussian noise. $\sigma_{\text {new }}$ is the new error for the new flux. We degraded all the spectra in each bin individually before we ran the co-addition. We remeasure all the chemical abundances described in Sections 3.4 and 3.5 but with the degraded spectra. Figures 18 and 19 present the comparison of $[\mathrm{Fe} / \mathrm{H}]$ and $[\alpha / \mathrm{Fe}]$ for GCs, respectively. For comparison purpose, we also plot the HRS metallicities of GCs in red. M5 gives the best match, and NGC 2419 shows a discrepancy in which the co-added metallicity is less than that determined from HRS by around 0.3 dex. $[\alpha / \mathrm{Fe}]$ shows larger scatter compared with the $[\alpha / \mathrm{Fe}]$ measured from spectra without degrading, especially for $[\mathrm{Mg} / \mathrm{Fe}]$. The comparisons for dSphs are shown in Figures 20 and 21. The bias trends are larger than the trend in Figure 8 in which the co-added degraded spectra tend to give metal-poorer results for the metalrich weighted-average groups but the inverse is true for the metal-poor weighted-average groups. The matches are good, however, for both binning approaches. The $[\alpha / \mathrm{Fe}]$ measured from degraded spectra with low $\mathrm{S} / \mathrm{N}$ like in M31 satellite RGB stars does reflect the mean value of these bins of stars. [Ti/Fe] shows a good match while the limited neutral lines for $\mathrm{Mg}$ with such low $\mathrm{S} / \mathrm{N}$ make the accuracy worse.

We acknowledge that the procedure we are using to degrade the Milky Way GC and dSph spectra does not exactly mimic the situation with the co-addition of the low-S/N M31 spectra. The Milky Way spectra were degraded after sky subtraction and continuum division, whereas these steps may be more difficult for the M31 spectra given their low S/N.

\subsection{Discussion}

The discrepancy between the co-added and weighted-average abundances indicates the intrinsic degeneracy in stellar parameter space, and it is hard for us to disentangle this degeneracy just using our current data sets. Uncertainties in the measurements also can cause discrepancy. Understanding causes of the bias is essential for future abundance analysis once we apply the co-addition technique to RGB stars in the Andromeda system, for this discrepancy will surely be present in those abundance measurements. We also rate the two binning scenarios in this part. 


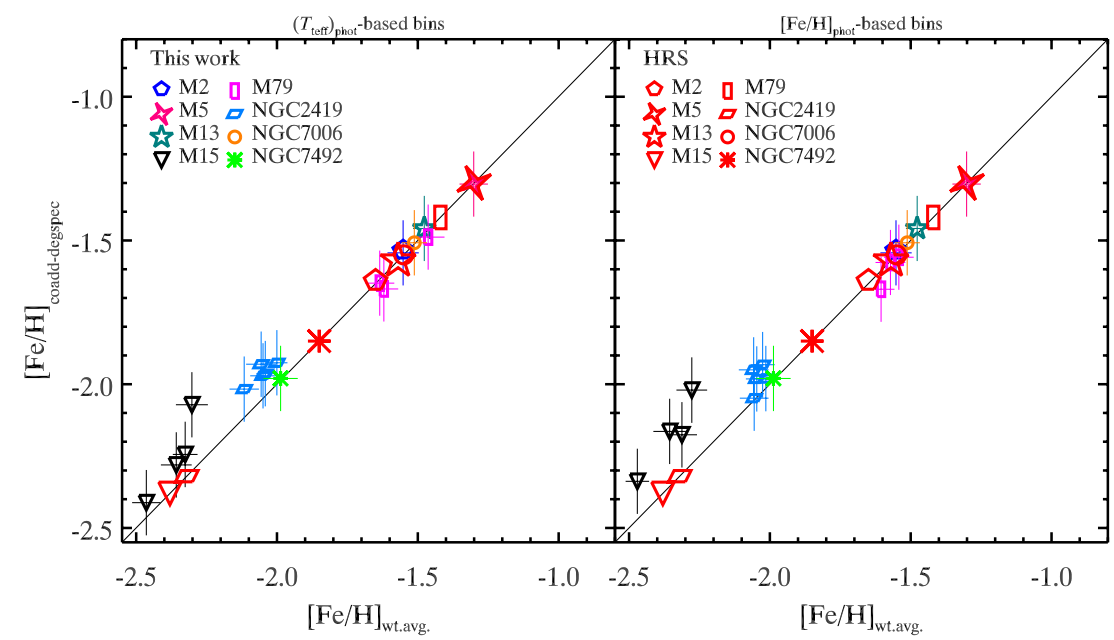

Figure 18. Comparison between weighted-average metallicity and metallicity from co-added degraded spectra for eight GCs in the Milky Way. The value of the vertical axis represents co-added results and horizontal axis is weighted-average results. For weighted-average abundances, we measured every star's abundance and averaged the abundances, weighting by the elemental mask weights which we used in the combining of individual spectrum. We selected stars with log $g \leqslant 1.4$ for all eight GC stars and binned stars by photometric effective temperature (left) and photometric metallicity (right), respectively. The symbols are same as in Figure 6. The symbols in red are the metallicities measured by high-resolution spectroscopy (HRS). M2 (NGC 7089): Harris catalog (http://physwww.physics.mcmaster.ca/ harris/mwgc.dat). M5 (NGC 5904), M13 (NGC 6205), M15 (NGC 7078), M79 (NGC 1904), NGC 2419, NGC 7006, and NGC 7492: Pritzl et al. (2005). Figure 6 presents the same information based on non-degraded spectra with the observed $\mathrm{S} / \mathrm{N}$.

(A color version of this figure is available in the online journal.)

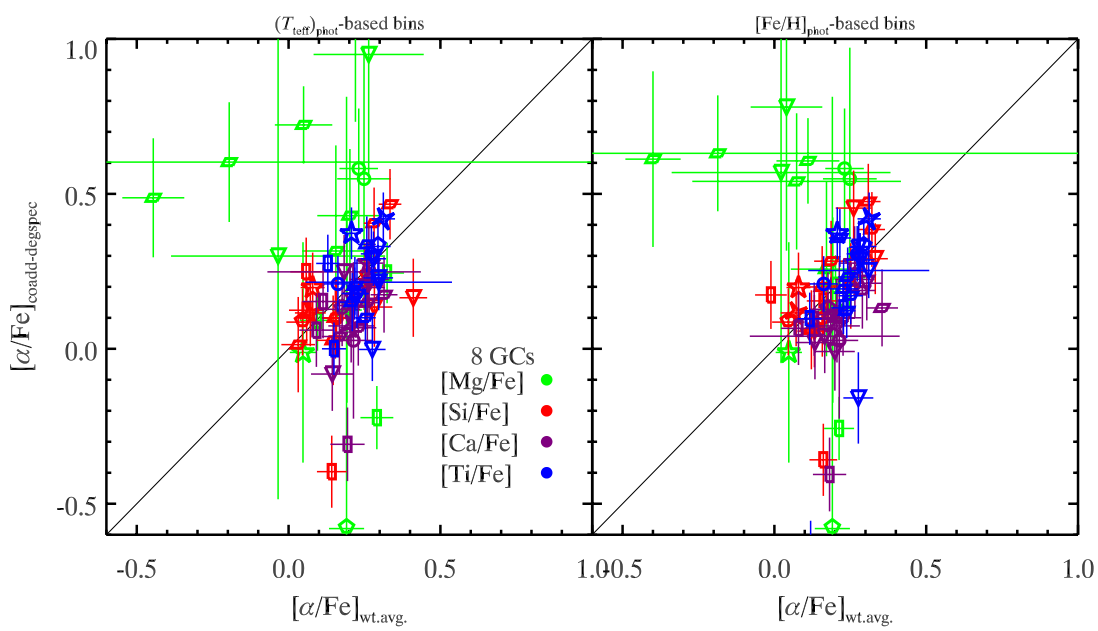

Figure 19. Same as Figure 18 except for $[\alpha / \mathrm{Fe}]$ instead of $[\mathrm{Fe} / \mathrm{H}]$. The different symbols denote the different GCs as defined in Figure 18 but with different colors for the different $\alpha$-elements. $[\mathrm{Mg} / \mathrm{Fe}]$ in green, $[\mathrm{Si} / \mathrm{Fe}]$ in red, $[\mathrm{Ca} / \mathrm{Fe}]$ in purple, and $[\mathrm{Ti} / \mathrm{Fe}]$ in blue. Figure 7 presents the same information based on non-degraded spectra with the observed $\mathrm{S} / \mathrm{N}$.

(A color version of this figure is available in the online journal.)

\subsection{1. $[\mathrm{Fe} / \mathrm{H}]$}

Both GCs and dSphs display trends in which measured metallicities from co-added spectra are likely to be higher than the weighted-average metallicities, except for NGC 7492 and Fornax. GCs are simple stellar systems with old age and a single metallicity. In our GCs sample, M15, M79, and NGC 2419 have more than one bin and these bins show a scatter covering an expanded range in $\left\langle\mathrm{S} / \mathrm{N}_{\text {coadd }}\right\rangle,\left\langle T_{\text {eff }}\right\rangle$, and $\langle\log g\rangle$ in two binning approaches. The $\left\langle T_{\text {eff }}\right\rangle$ shows more impact on the scatter, which increasing $T_{\text {eff }}$ result bigger scatter. The rest of GCs with one bin show a good match between co-add and weighted-average results. The $\mathrm{S} / \mathrm{Ns}$ for GCs are too high to assess its impact in Figure 11, but for the degraded spectra of GCs, Figure 18 demonstrates that even for spectra having $\mathrm{S} / \mathrm{N}$ as low as 10 pixel $^{-1}$, the co-addition technique can still produce reliable measurements, and the differences between coadded metallicities and HRS metallicities are acceptable. For the scatter of bins of M15, M79, and NGC 2419, we surmise the main reason is measurement uncertainty.

The dSphs have more complicated and extended star formation histories with spreads in their metallicity distributions. Like GCs, $\mathrm{S} / \mathrm{N}$ does not seem to be a dominant factor in the measurement bias for both high-S/N co-added spectra in the top panels of Figure 12 and degraded co-added spectra in Figure 20. The mean effective temperature, as Figure 14 (top panels) shows, is tightly related to the metallicity residuals, especially for $T_{\text {eff }} \geqslant 4300 . \log g$ seems to have a slight impact on the residuals of stars binned by $T_{\text {eff }}$ as Figure 16 shows, which just demonstrates what we note in Section 3.4, which is that our synthetic spectral measurements use neutral metal lines which are insensitive to surface gravity. 


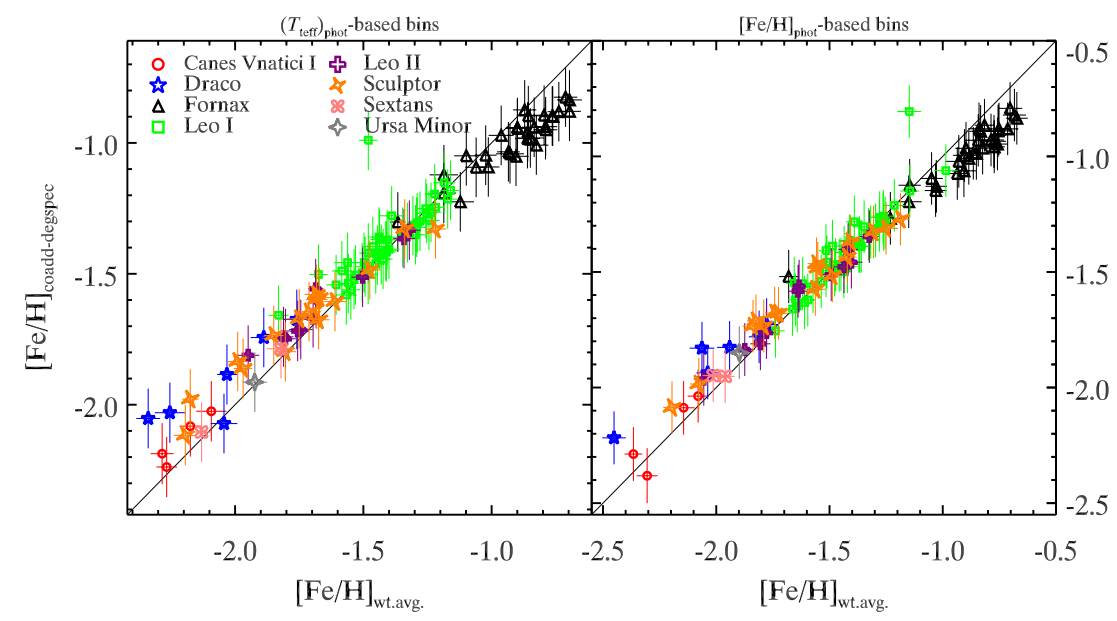

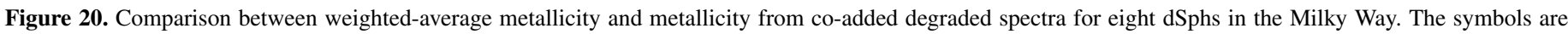

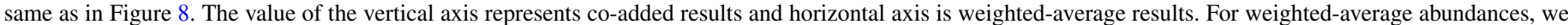

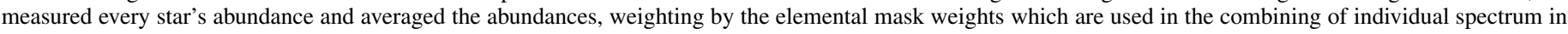

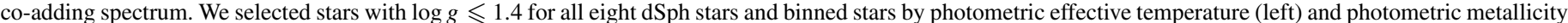
(right), respectively. Figure 8 presents the same information based on non-degraded spectra with the observed S/N.

(A color version of this figure is available in the online journal.)

The detailed comparisons of medium-resolution results and HRS results for individual stars of dSphs can be found in Kirby et al. (2010).

Effective temperature has an important impact on the trends of residuals. Metallicities determined from the co-added spectra of metal-rich stars with low effective temperature (e.g., Fornax) tend to be more metal-poor compared with metallicities derived from the weighted-average method. As the effective temperature increases, the metallicity measurement residuals for co-added spectra become more metal-rich. In spectral synthesis, when $T_{\text {eff }}$ increases, it affects $[\mathrm{Fe} / \mathrm{H}]$ because more metals are needed in the synthetic atmosphere to compensate for the weakening lines caused by increasing temperature (KGS08), and vice versa for metal-rich stars with lower effective temperature. In spectral co-addition, this effect seems to be amplified, probably because there is a range of effective temperature for each bin, and the stars with higher effective temperature favor the more metal-rich synthetic one to compensate the temperatureinduced weakening lines, and vice versa. Schlaufman et al. (2011) noticed the effect of the effective temperature on the co-addition method, and they employed $500 \mathrm{~K}$ bins in their stellar co-additions. Our bins of stars do not have a fixed range in effective temperature but $T_{\text {eff }}$ for each bin are in close proximity, a range smaller than $500 \mathrm{~K}$, as shown in the left panels of Figure 3. Since $T_{\text {eff }}$ and $\log g$ are correlated, we expect $\log g$ would have the same impact on metallicity, but it shows a less prominent impact than $T_{\text {eff }}$ on metallicity. The intrinsic metallicity dispersion of individual systems also plays an essential role in the individual discrepancy of different stellar systems. Schlaufman et al. (2011) determined the average metallicity of M13 and M15 by co-addition but using metal-poor main-sequence turnoff stars from the Milky Way halo. At lower spectral resolution, they measured $[\mathrm{Fe} / \mathrm{H}]=-1.7 \pm 0.15$ for $\mathrm{M} 13$ and $[\mathrm{Fe} / \mathrm{H}]=-2.4 \pm 0.2$ for M15. Our results are more accurate, and the bias is less than 0.01 for M13 and less than 0.06 for M15 (Figure 6).

$$
\text { 4.4.2. }[\alpha / F e]
$$

Compared to the iron abundance, determining individual $\alpha$-element abundances is more difficult, especially for distant stars with low $\mathrm{S} / \mathrm{N}$. For both high-S/N spectra and degraded spectra, the comparisons of $[\alpha / \mathrm{Fe}]$ between co-added and weighted-average results show conspicuous scatter, especially for $[\mathrm{Mg} / \mathrm{Fe}]$. For GCs, the most biased bins are from the metalpoor systems M15 and NGC 2419, as Figures 7 and 19 show, indicating that the measurements of $\alpha$-elements, particularly $[\mathrm{Mg} / \mathrm{Fe}]$, are very sensitive to metallicity. Increasing $\left\langle T_{\text {eff }}\right\rangle$ still has an impact on the scatter in multi-bin GCs. M13, with the lowest $\left\langle T_{\text {eff }}\right\rangle$, presents an obvious bias in $[\mathrm{Ca} / \mathrm{Fe}]$ and $[\mathrm{Ti} / \mathrm{Fe}]$. Compared to $[\mathrm{Mg} / \mathrm{Fe}]$, however, these biases are still acceptable, even for the results derived from degraded spectra. $\mathrm{S} / \mathrm{N}$ plays a more important role in $\alpha$ measurements, particular for $[\mathrm{Mg} / \mathrm{Fe}]$ and $[\mathrm{Ti} / \mathrm{Fe}$ (Figure 11). The same impact of $\mathrm{S} / \mathrm{N}$ also shown in Figure 12 for dSphs. Another main reason for the scatter is the measurement uncertainty, especially for $[\mathrm{Mg} / \mathrm{Fe}]$ which can be seen in the measurements of degraded spectra (Figure 19). For dSphs, the number of bins makes the impacts of different parameters on the residual biases more clear. [Si/Fe], $[\mathrm{Ca} / \mathrm{Fe}]$, and $[\mathrm{Ti} / \mathrm{Fe}]$ show good agreement between co-addition and weighted-average abundances in Figure 9. We suspect that the large scatter for Leo I results from the low $\mathrm{S} / \mathrm{N}$ and relatively high and extended coverage in $\left\langle T_{\text {eff }}\right\rangle$. For the outliers, CVnI, Sculptor, and Sextans, uncertainty in photometrically estimating $T_{\text {eff }}$ and $[\mathrm{Fe} / \mathrm{H}]$, which we used to bin the stars, is another possible biasing source (Figure 17). Comparing Figures 12, 14, and 16 , it seems that $\mathrm{S} / \mathrm{N}, T_{\text {eff }}$, and $\log g$ are all possible reasons for the residual biases, especially for stars binned by $\left(T_{\text {eff }}\right)_{\text {phot }}$. Digging deeper, $\log g$ is a function of stellar mass and radius, but the mass and radius of star are also related to $T_{\text {eff }}$. If $[\mathrm{Fe} / \mathrm{H}]$ is fixed, increasing $T_{\text {eff }}$ will make absorption lines more shallow and narrow, but increasing $\log g$ will broaden these lines. If we consider variation in $[\mathrm{Fe} / \mathrm{H}]$, then it becomes even more difficult to distinguish which matters most. These small features affect the abundance measurements for both individual and co-added spectra. Although stars in each bin have similar properties, the exact values of these important stellar parameters are different. We used the same chemical abundances to select the synthetic spectra for each bin, but the photometric $T_{\text {eff }}$ and $\log g$ values used were those of individual observed stars. Considering the sensitivity of $\alpha$-elements to the metallicity, this assumption 


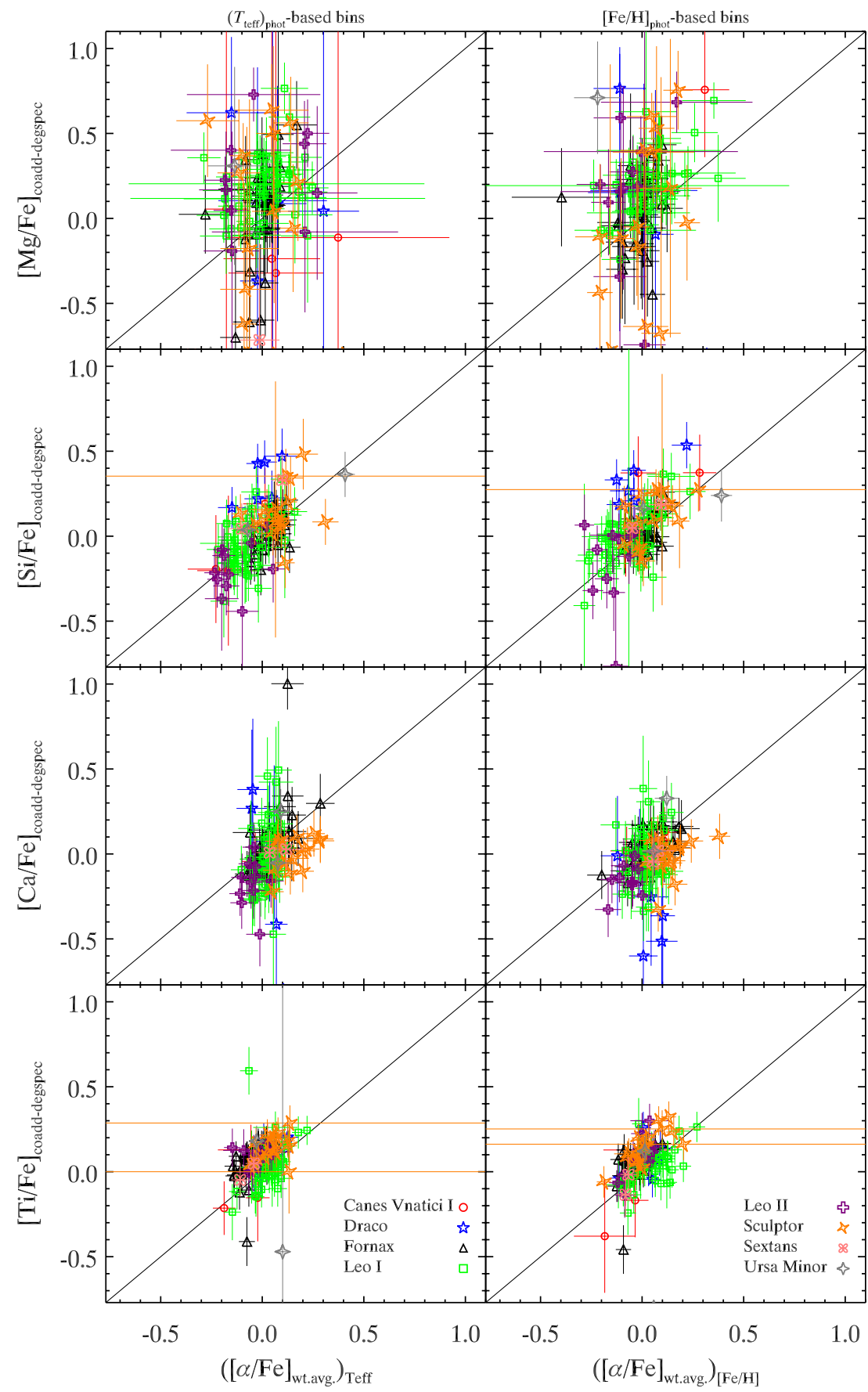

Figure 21. Comparison for $\alpha$-element abundances between weighted-average abundances and co-added degraded spectral abundances for four $\alpha$-elements, Mg, Si, $\mathrm{Ca}$, and $\mathrm{Ti}$ (top to bottom). Stars used for these plots are from the eight dSph galaxies. Points in the left panels are stars binned by $T_{\text {eff }}$ and in the right panels are binned by $[\mathrm{Fe} / \mathrm{H}]_{\text {phot }}$. The symbols are same as in Figure 20. Figure 9 presents the same information based on non-degraded spectra with the observed S/N.

(A color version of this figure is available in the online journal.)

may play a role in the large scatter of $[\alpha / \mathrm{Fe}]$. Compared to other $\alpha$-elements, $[\mathrm{Mg} / \mathrm{Fe}]$ is the most difficult one to measure accurately because of weak absorption lines. Magnesium is a product of SN II, and it is the least visible of the $\alpha$-elements in the DEIMOS spectra (KGS08). The elemental mask for $[\mathrm{Mg} /$ $\mathrm{Fe}]$ contains a limited number of absorption lines compared with other elemental masks, therefore results in a larger uncertainty for $[\mathrm{Mg} / \mathrm{Fe}]$.

All eight dSphs in this work have significant intrinsic spread in metallicity (Figure 8). The intrinsic spread in the abundance distribution of dwarf satellite galaxies indicates their extended star formation history (Venn et al. 2004; Helmi et al. 2006; Cohen \& Huang 2009; Kirby et al. 2009). The target selection of a stellar system which has radial metallicity gradient like Sculptor (Tolstoy et al. 2004; Walker et al. 2009; Kirby et al. 2009) may influence the measured abundance distribution for the co-added spectra method. Therefore, when we apply coaddition to dSphs, the effect of a metallicity spread on the $\alpha$ elements abundance measurements with limited absorption lines need to be considered carefully. Particularly for the stars in M31 satellite galaxies with low S/N, Figures 19 and 21 show similar scatter amplitudes, demonstrating that we can estimate the mean 


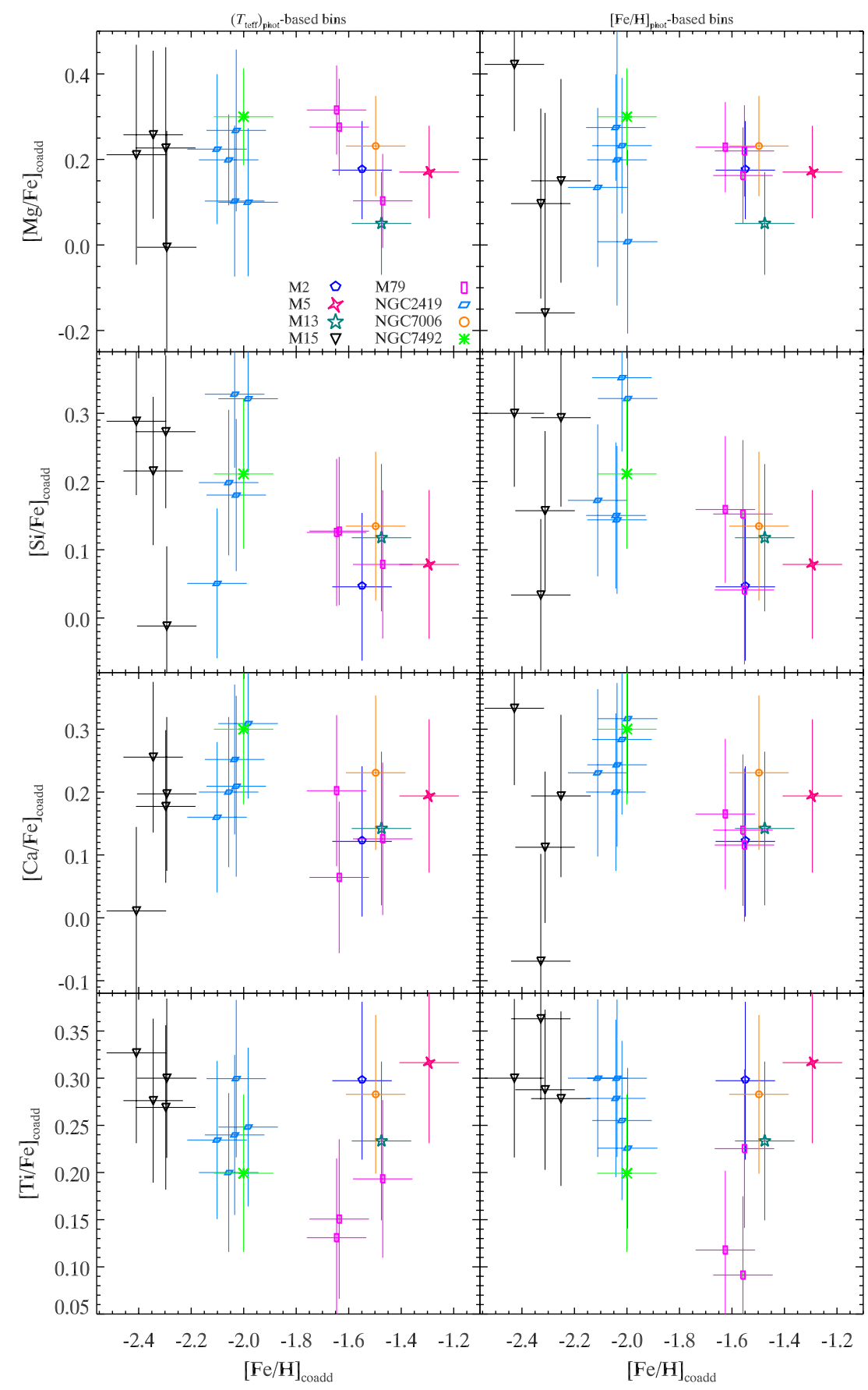

Figure 22. Element abundances for eight GCs from co-added spectra. The left panels represent results binned by photometric effective temperature and the right panels are binned by photometric metallicity.

(A color version of this figure is available in the online journal.)

values and trends for $\alpha$-elements from low-S/N spectra with the co-addition technique.

\subsection{3. $[\alpha / \mathrm{Fe}]$ versus $[\mathrm{Fe} / \mathrm{H}]$}

Figures 22 and 23 present the individual $\alpha$-element distribution versus metallicity determined from co-added spectra for eight GCs and eight dSphs, respectively. The trends are clearly shown for the majority of bins, and only Leo I and Fornax display a very blurred trend in $[\mathrm{Ca} / \mathrm{Fe}]$ distribution. We add individual stars' abundances in the figures as Figure 24 shows. The distributions of [Ti/Fe] from Leo I and Fornax seem to display an upward tendency with increasing metallicity compared with previous results shown by individual stars (Kirby et al. 2011a, their Figure 13). We speculate that it is mainly because our surface gravity restriction has removed fainter RGB stars, which are located in the metal-rich region in Figure 23. Yet, we still consider the co-added spectra method as an efficient and feasible tool to proceed the detailed multi-element abundance measurement with medium-resolution spectra. Based on the detailed $\alpha$-element abundance analysis (Shetrone et al. 2001, 2003; Venn et al. 2004; Kirby et al. 2011a), we expect to extend our understanding and insight into the star formation history and galaxy evolution to the Andromeda galaxy system and even farther systems with this technique. 


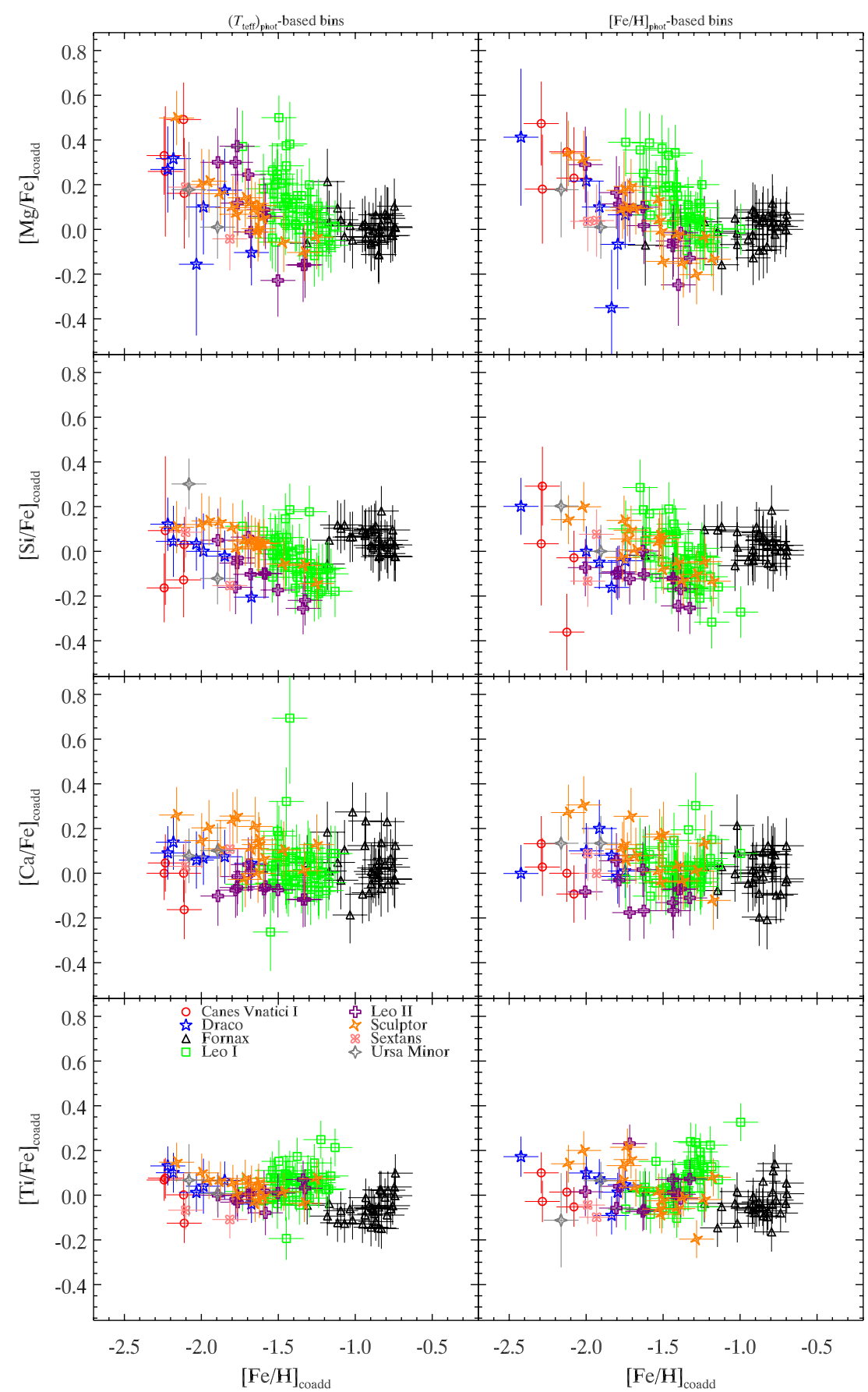

Figure 23. Multi-element abundances for eight dSphs from co-added spectra. The left panels represent results binned by photometric effective temperature and the right panels are binned by photometric metallicity. The symbols are the same as in Figure 8.

(A color version of this figure is available in the online journal.)

\subsubsection{Binning Scenarios}

By comparing two binning algorithms, we can assess which one better reflects the true values of groups of stars. The two parameters we used to bin, $T_{\text {eff }}$ and $[\mathrm{Fe} / \mathrm{H}]_{\text {phot }}$, are primarily estimated from isochrone fitting on CMD. Stellar parameters estimated from photometry are far less accurate than the ones derived by spectroscopy. On the other hand, some assumptions must be made to constrain the parameter space in the CMD. Here we have assumed all RGB stars have an age of $14 \mathrm{Gyr}$ for both GCs and dSphs. For the dSph galaxies, this assumption is too simple because some dSphs are known to have extended star formation histories compared with GCs (Harris 2001; Puzia 2003; Harris et al. 2006). Sculptor and Sextans are dominated by old populations (Orban et al. 2008), which should show observational properties similar to Galactic GCs. However, Shetrone et al. (2003) proved that Sculptor has a spread in age of at least 4 Gyr. They also demonstrated that Fornax has a complicated star formation history from $15 \mathrm{Gyr}$ to $0.5 \mathrm{Gyr}$ ago, and Carina and Leo I have an age span of 4-7 Gyr and 2-7 Gyr, respectively. de Boer et al. (2012) combined deep photometry with HRS to study the detailed star formation history 


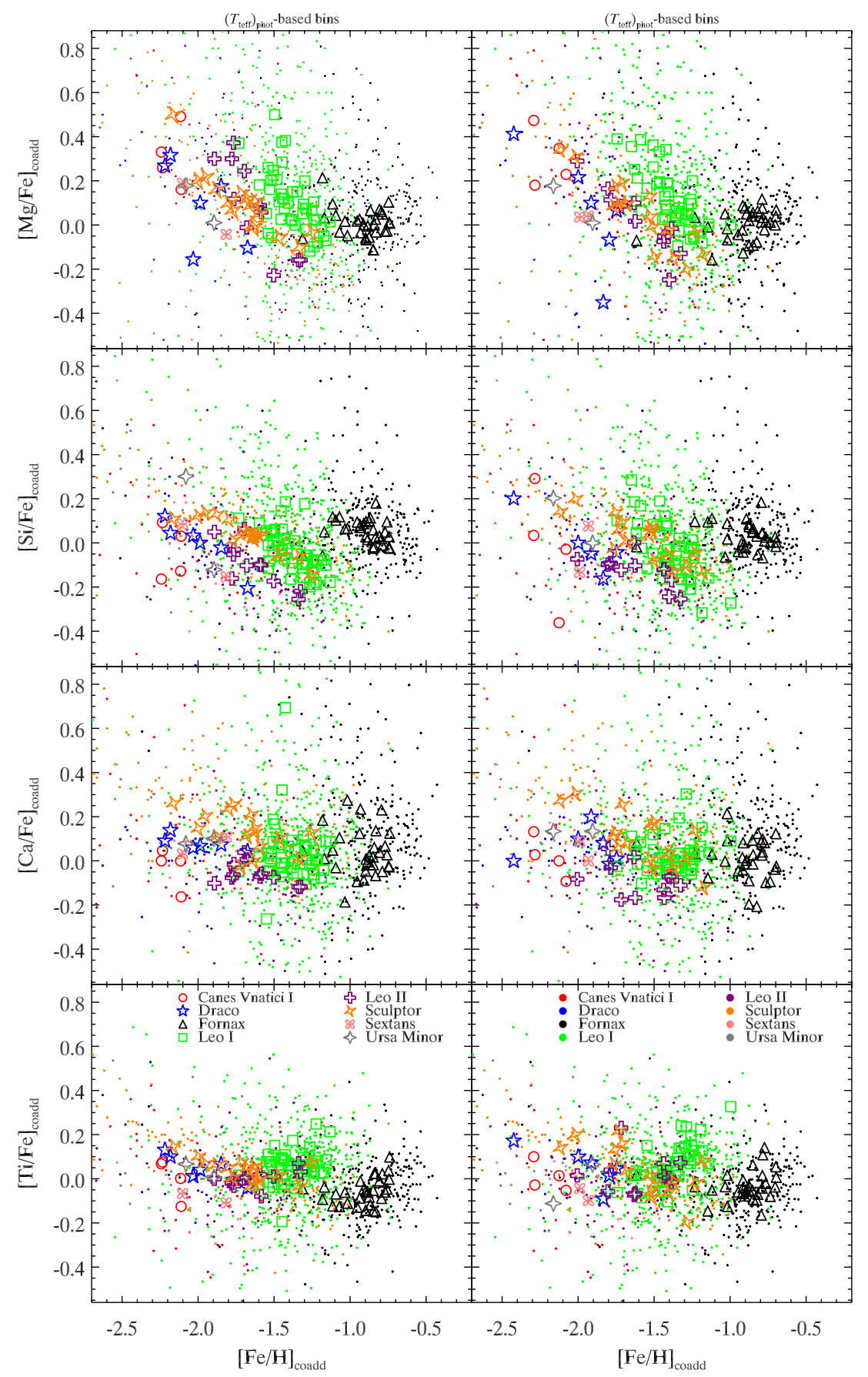

Figure 24. Same as Figure 23, but abundances of individual stars in each bin have been added in (the colorful points).

(A color version of this figure is available in the online journal.)

and estimate ages for individual RGB stars in the Sculptor. They found that old ( $>10 \mathrm{Gyr}$ ) metal-poor stars, and younger, more metal-rich populations are both present in Sculptor. By finding a knee in the $\alpha$-element distribution at age around 10.9 Gyr they surmised that SN Ia enrichment began ( $2 \pm 1 \mathrm{Gyr})$ after the beginning of star formation in Sculptor. Furthermore, comparisons of the metallicities of the Milky Way dSph galaxies derived from spectroscopy versus photometry have indicated a discrepancy between the two techniques (Lianou et al. 2011). Lianou et al. (2011) demonstrated that the assumption of old ages for RGB stars in dwarf satellite galaxies leads to a bias toward metal-poor estimates if the ages of the stars are lower, as is possibly the case for Fornax and Leo II. The ages of stars are always difficult to estimate, although one technique is based on using the spectroscopic metallicity, and then measuring the age using photometry (KGS08).

In this work, the age affect is not enough to highly bias our results, despite the age-spread in dSphs. In order to test the impact of age, we have tried three ages in our photometric metallicity determination, $7 \mathrm{Gyr}, 9 \mathrm{Gyr}$, and $14 \mathrm{Gyr}$, and we found no significant difference in these ages. Then we choose 14 Gyr to set to all the test RGB stars and make comparison of metallicity derived from co-added spectra and isochrone fitting photometrically for GCs and dSphs, as shown in Figure 17. 
There is a good reason, however, that $[\mathrm{Fe} / \mathrm{H}]_{\text {phot }}$ is less than $[\mathrm{Fe} / \mathrm{H}]_{\text {coadd }}$ for Fornax. The average age is significantly younger than $14 \mathrm{Gyr}$, which means the RGB stars are bluer than the $14 \mathrm{Gyr}$ isochrone. So $[\mathrm{Fe} / \mathrm{H}]_{\text {phot }}$ will be too low when we assume the age is 14 Gyr. The uncertainty in $[\mathrm{Fe} / \mathrm{H}]_{\text {phot }}$ may affect the results if $[\mathrm{Fe} / \mathrm{H}]_{\text {phot }}$ is significantly different from $[\mathrm{Fe} / \mathrm{H}]$ determined by spectroscopy. However, binning by $[\mathrm{Fe} / \mathrm{H}]_{\text {phot }}$ is still efficient, and Figure 8 shows that the span of metallicities derived from stars binned by $[\mathrm{Fe} / \mathrm{H}]_{\text {phot }}$ covers a larger range than the one binned by $T_{\text {eff }}$, meaning that we would most likely discover extremely metal-poor stars by this way in more distant stellar systems. These intrinsic extremely metal-poor stars play a crucial role in chemical evolution of these satellite galaxies and accretion history of galactic stellar halo (Tolstoy et al. 2001; Helmi et al. 2006; Kirby et al. 2008b).

In the parameter space, the residuals of abundance binned by $T_{\text {eff }}$ span a larger range, as shown in Figures 14 and 16, illustrating that these RGB stars are very sensitive to $T_{\text {eff }}$ and show a larger variance with parameters such as $\mathrm{S} / \mathrm{N}, T_{\text {eff }}$, and $\log g$. However, the standard deviations of distributions show that chemical abundances determined by $[\mathrm{Fe} / \mathrm{H}]_{\text {phot }}$-binned stars are tighter and similar to the weighted-average abundances. The outliers in the $T_{\text {eff }}$-binned stars are expected to induce the main source of the large scatter. In the future, we expect to combine these two parameters together, and to explore the impact of relationship between ages, photometric $T_{\text {eff }}$ and $\log g$, and metallicity on binning stars. Disentangling this problem will decrease the scatter and improve the agreement between co-added results and weighted-average abundances.

\section{SUMMARY AND APPLICATIONS}

In this paper, we present a method for spectral co-addition of medium-resolution spectra for detailed measurements of multielement abundances. Our method overcomes the low-S/N and weak spectral features of distant RGB stars by co-adding spectra of similar RGB stars. We impose a surface gravity restriction $(\log g \leqslant 1.40)$ for RGB stars in GCs and dSphs in order to enhance the quality of spectra. We group stars in two ways, using photometric effective temperature estimates and photometric metallicity estimates, and then we determine detailed chemical abundances $([\mathrm{Fe} / \mathrm{H}],[\mathrm{Mg} / \mathrm{Fe}],[\mathrm{Si} / \mathrm{Fe}],[\mathrm{Ca} / \mathrm{Fe}],[\mathrm{Ti} / \mathrm{Fe}])$ with elemental masks on the whole spectrum. We use more than 1300 well-studied individual RGB stars from eight GCs and eight dSphs in the Milky Way to test the feasibility and accuracy of this method. This work can briefly be summarized as follows.

First, we determine effective temperature, surface gravity, and iron abundance by an isochrone-fitting technique on the CMD for individual RGB stars in our sample, then set these parameters as initial values for the following selection of synthetic spectra for each observed one and the chemical abundance comparisons. Then, individual chemical abundances for all RGB stars have been determined and compare well with previous results (KGS08; Kirby et al. 2009, 2010).

Second, we make a cut $(\log g \leqslant 1.4)$ on candidate RGB stars so that our resulting sample is a good match to the M31 red giant stars to which we aim to apply this spectral co-addition method. Then the candidate stars were binned using two photometric parameters, effective temperature and metallicity. The science spectra belonging to one bin were added together, weighted by the inverse variance within specific elemental masks on the pixel level. We also selected a synthetic spectrum for each observed spectrum in each bin based on its photometric effective temperature and photometric surface gravity. The selected synthetic spectra have the same metallicity and $\alpha$-element abundances for each bin. Then, we added these synthetic candidate spectra together with the same weights as we used for the observed ones.

Third, we used the Levenberg-Marquardt algorithm to select the best-fitting co-added synthetic spectrum for the co-added observed one by minimizing $\chi^{2}$ between synthetic one and observed one for whole spectrum on a pixel-to-pixel level in several iteratively steps. For each bin we determine each elemental abundance, $[\mathrm{Fe} / \mathrm{H}],[\mathrm{Mg} / \mathrm{Fe}],[\mathrm{Si} / \mathrm{Fe}],[\mathrm{Ca} / \mathrm{Fe}],[\mathrm{Ti} / \mathrm{Fe}]$, separately. Only one element was considered in each run excluding bias probably induced by unavailable element abundances of individual stars.

Fourth, we combined individual abundances in each bin weighted almost the same way as we performed to the coadded spectra, to get the weighted-average abundances, and then we carried out comparisons with co-added abundances. The co-added abundances agree reasonably well with the expected values from the weighted-average abundances.

Fifth, for $\left(T_{\text {eff }}\right)_{\text {phot }^{-}}$versus $[\mathrm{Fe} / \mathrm{H}]_{\text {phot }}$-based binning scenarios, the abundance comparison and difference plots look similar for the two scenarios. This means the abundance errors are dominated by factors other than the spread of absorption line strength within a bin. For metal-poor stars, the RGB isochrones are really vertical so the two binning scenarios result in similar groups of stars. We prefer the $[\mathrm{Fe} / \mathrm{H}]_{\text {phot }}$-based binning scenario since our co-addition scenario for synthetic spectra can account for a spread in $T_{\text {teff }}$ within a bin, but not for a spread in $[\mathrm{Fe} / \mathrm{H}]$ or $[\alpha / \mathrm{Fe}]$.

Last, the precision of the abundances measured from coadded medium-resolution spectrum does not appear to be a strong function of $\mathrm{S} / \mathrm{N}$ over the range we have explored: co-added spectral $\mathrm{S} / \mathrm{N} \sim 200-2000 \mathrm{pixel}^{-1}$ for GCs (see Figure 11) and $\mathrm{S} / \mathrm{N} \sim 50-300 \mathrm{pixel}^{-1}$ for $\mathrm{dSphs}$ (see Figure 12). The $\mathrm{S} / \mathrm{N}$ of the individual M31 RGB spectra to which we plan to apply this co-addition method is typically much lower than that of the individual spectra analyzed in this paper, but many more stars are co-added together in the M31 bins so that the $\mathrm{S} / \mathrm{N}$ of the co-added M31 RGB spectra are expected to be comparable to that of the co-added spectra used here (Kirby et al. 2010). The comparisons of metallicity and $\alpha$-elements derived from the degraded spectra with $\mathrm{S} / \mathrm{N}$ comparable to RGB stars of M31 satellite galaxies and weighted-average results demonstrate the feasibility of this technique.

We conclude that we can safely apply this method of spectral co-addition to analyze chemical abundance patterns in M31 satellite galaxies using medium-resolution spectra of RGB stars. This will be a provide a useful start for detailed chemical abundance exploration beyond the Milky Way system.

L.Y. and E.W.P. gratefully acknowledge partial support from the Peking University Hundred Talent Fund (985) and grants 10873001 and 11173003 from the National Natural Science Foundation of China (NSFC). L.Y. also acknowledges support from the LAMOST-PLUS collaboration, a partnership funded by NSF grant AST-09-37523, and NSFC grants 10973015 and 11061120454.

Support for this work was also provided by NASA through Hubble Fellowship grant 51256.01 awarded to E.N.K. by the Space Telescope Science Institute, which is operated by the Association of Universities for Research in Astronomy, Inc., for NASA, under contract NAS 5-26555. 
P.G. acknowledges support from NSF grant AST-10-10039. He thanks the staff of the Kavli Institute for Astronomy and Astrophysics at Peking University for their generous hospitality during his collaborative visits.

L.C. was supported by UCSC's Science Internship Program (SIP).

\section{Facility: Keck:II (DEIMOS)}

\section{REFERENCES}

Adelman, S. J., \& Leckrone, D. S. 1985, in Horizontal-Branch and UV Bright Stars, ed. A. G. D. Philip (New York: L. Davis), 75

Anders, E., \& Grevesse, N. 1989, GeCoA, 53, 197

Armandroff, T. E., Da Costa, G. S., Caldwell, N., \& Seitzer, P. 1993, AJ, 106, 986

Armandroff, T. E., \& Zinn, R. 1988, AJ, 96, 92

Beers, T. C., Rossi, S., Norris, J. E., Ryan, S. G., \& Shefler, T. 1999, AJ, 117,981

Bica, E., \& Alloin, D. 1987, A\&A, 186, 49

Castelli, F. 2005, MSAIS, 8, 34

Castelli, F., Grantton, R. G., \& Kurucz, R. L. 1997, A\&A, 318, 841

Castelli, F., \& Kurucz, R. L. 2004, arXiv:astro-ph/0405087

Choi, P. I., Guhathakurta, P., \& Johnston, K. V. 2002, AJ, 124, 310

Cohen, J. G., \& Huang, W. 2009, ApJ, 701, 1053

Cole, S., Lacey, C. G., Baugh, C. M., \& Frenk, C. S. 2000, MNRAS, 319, 168

Collins, M. L. M., Chapman, S. C., Irwin, M. J, et al. 2010, MNRAS, 407, 2411

Colucci, J. E., Bernstein, R. A., Cameron, S., McWilliam, A., \& Cohen, J. G. 2009, ApJ, 704, 385

Cooper, M. C., Newman, J. A., Davis, M., Finkbeiner, D. P., \& Gerke, B. F. 2012, spec2d, ascl soft, 1203.003

Da Costa, G. S., Armandroff, T. E., \& Caldwell, N. 2002, AJ, 124, 332

Da Costa, G. S., Armandroff, T. E., Caldwell, N., \& Seitzer, P. 1996, AJ, 112,2576

Da Costa, G. S., Armandroff, T. E., Caldwell, N., \& Seitzer, P. 2000, AJ, 119,705

de Boer, T. J. L., Tolstoy, E., Hill, V., et al. 2012, A\&A, 539, A103

Demarque, P., Woo, J.-H., Kim, Y.-C., \& Yi, S. K. 2004, ApJS, 155, 667

Diemand, J., Kuhlen, M., \& Madau, P. 2007, ApJ, 667, 859

Faber, S. M., Phillips, A. C., Kibrick, R. I., et al. 2003, Proc. SPIE, 4841, 1657

Foster, C., Forbes, D. A., Proctor, R. N., et al. 2010, AJ, 139, 1566

Frayn, C. M., \& Gilmore, G. F. 2002, MNRAS, 337, 445

Gallazzi, A., Brinchmann, J., Charlot, S., \& White, S. D. M. 2008, MNRAS, 383,1439

Gilmore, G. 2004, Sci, 304, 1915

Girardi, L., Bertelli, G., Bressan, A., et al. 2002, A\&A, 391, 195

Grebel, E. K., \& Gallagher, J. S., III. 2004, ApJ, 610, 89

Greggio, L., \& Renzini, A. 1983, MmSAI, 54, 311

Guhathakurta, P., Beaton, R., Bullock, J., et al. 2010, A\&AS, 42, 537

Guhathakurta, P., Rich, R. M., Reitzel, D. B., et al. 2006, AJ, 131, 2497

Harris, G. L. H., \& Harris, W. E. 2000, AJ, 120, 2423

Harris, G. L. H., Harris, W. E., \& Poole, G. B. 1999, AJ, 117, 855

Harris, W. E. 2001, in Star Clusters, ed. L. Labhardt \& B. Binggeli (Berlin: Springer), 223

Harris, W. E., Whitmore, B. C., Karakla, D., et al. 2006, ApJ, 636, 90

Helmi, A., Irwin, M. J., Tolstoy, E., et al. 2006, ApJL, 651, L121

Holberg, J. B., Barstow, M. A., Hubeny, I., et al. 2003, in ASP Conf. Ser. 291, Hubble's Science Legacy: Future Optical/Ultraviolet Astronomy from Space, ed. K. R. Sembach, J. C. Blades, G. D. Illingworth, \& R. C. Kennicutt, Jr. (San Francisco, CA: ASP), 383

Ibata, R. A., Irwin, M., Lewis, G., Ferguson, A. M. N., \& Tanvir, N. 2001, Natur, 412,49

Ishigaki, M. N., Chiba, M., \& Aoki, W. 2012, ApJ, 753, 64

Kalirai, J. S., Beaton, R. L., Geha, M. C., et al. 2010, ApJ, 711, 671

Kalirai, J. S., Zucker, D. B., Guhathakurta, P., et al. 2009, ApJ, 705, 1043

Kirby, E. N. 2011, PASP, 123, 531

Kirby, E. N., Cohen, J. G., Smith, G. H., et al. 2011a, ApJ, 727, 79

Kirby, E. N., Guhathakurta, P., Bolte, M., Sneden, C., \& Geha, M. C. 2009, ApJ, 705,328
Kirby, E. N., Guhathakurta, P., Simon, J. D., et al. 2010, ApJS, 191, 352

Kirby, E. N., Guhathakurta, P., \& Sneden, C. 2008a, ApJ, 682, 1217, (KGS08)

Kirby, E. N., Lanfranchi, G. A., Simon, J. D., Cohen, J. G., \& Guhathakurta, P. 2011b, ApJ, 727, 78

Kirby, E. N., Simon, J. D., Geha, M., Guhathakurta, P., \& Frebel, A. 2008b, ApJ, 685,43

Koch, A., Rich, R. M., Reitzel, D. B., et al. 2007, AN, 328, 653

Kupka, F., Piskunov, N., Ryabchikova, T. A., Stempels, H. C., \& Weiss, W. W. 1999, A\&AS, 138, 119

Kurucz, R. 1993, ATLAS9 Stellar Atmosphere Programs and 2 km/s Grid, Kurucz CD-ROM No. 13 (Cambridge, MA: Smithsonian Astrophysical Observatory)

Lanfranchi, G. A., \& Matteucci, F. 2004, MNRAS, 351, 1338

Letarte, B., Hill, V., Tolstoy, E., et al. 2010, A\&A, 523, A17

Lianou, S., Grebel, E. K., \& Koch, A. 2010, A\&A, 521A, 43

Lianou, S., Grebel, E. K., \& Koch, A. 2011, A\&A, 531A, 152L

Mannucci, F., Cresci, G., Maiolino, R., Marconi, A., \& Gnerucci, A. 2010, MNRAS, 408, 2115

Marble, A. R. 2004, Steward Obs.

Markwardt, C. B. 2009, in ASP Conf. Ser. 411, Astronomical Data Analysis Software and Systems XVIII, ed. D. A. Bohlender, D. Durand, \& P. Dowler (San Francisco, CA: ASP), 251

Matteucci, F., \& Recchi, S. 2001, ApJ, 558, 351

McConnachie, A. W., Irwin, M. J., Lewis, G. F., et al. 2004, MNRAS, 351, L94

Mouhcine, M., Rich, R. M., Ferguson, H. C., Brown, T. M., \& Smith, T. E. 2005, ApJ, 633, 828

Newman, J. A., Cooper, M. C., Davis, M., et al. 2012, ApJS, submitted (arXiv:1203.3192)

Olszewski, E. W., Schommer, R. A., Suntzeff, N. B., \& Harris, H. C. 1991, AJ, 101,515

Orban, C., Gnedin, O. Y., Weisz, D. R., et al. 2008, ApJ, 686, 1030

Pagel, B. E. J. 1997, Nucleosynthesis and Chemical Evolution of Galaxies (Cambridge: Cambridge Univ. Press)

Preston, G. W. 1961, ApJ, 134, 633

Pritzl, B. J., Venn, K. A., \& Irwin, M. 2005, AJ, 130, 2140

Puzia, T. 2003, in Extragalactic Globular Cluster Systems, ed. M. Kissler-Pating (Berlin: Springer), 267

Ramírez, I., \& Meléndez, J. 2005, ApJ, 626, 465

Reitzel, D. B., \& Guhathakurta, P. 2002, AJ, 124, 234

Rutledge, G. A., Hesser, J. E., \& Stetson, P. B. 1997, PASP, 109, 907

Saviane, I., Rosenberg, A., Piotto, G., \& Aparicio, A. 2000, A\&A, 355, 966

Sbordone, L. 2005, MSAIS, 8, 61

Sbordone, L., Bonifacio, P., Castelli, F., \& Kurucz, R. L. 2004, MSAIS, 5, 93

Schlaufman, K. C., Rockosi, C. M., Lee, Y. S., Beers, T. C., \& Allende Prieto, C. 2011, ApJ, 734, 49

Schlaufman, K. C., Rockosi, C. M., Lee, Y. S., et al. 2012, ApJ, 749, D77

Searle, L., \& Zinn, R. 1978, ApJ, 225, 357

Shetrone, M. D., Bolte, M., \& Stetson, P. B. 1998, AJ, 115, 1888

Shetrone, M. D., Cote, P., \& Sargent, W. L. W. 2001, ApJ, 548, 592

Shetrone, M. D., Siegel, M. H., Cook, D. O., \& Bosler, T. 2009, AJ, 137, 62

Shetrone, M. D., Venn, K. A., Tolstoy, E., et al. 2003, AJ, 125, 684

Sneden, C., Kraft, R. P., Prosser, C. F., \& Langer, G. E. 1992, AJ, 104, 2121

Sneden, C. A. 1973, PhD thesis, Univ. Texas at Austin

Strigari, L. E., Frenk, C. S., \& White, S. D. M. 2010, MNRAS, 408, 2364

Tanaka, M., Chiba, M., Komiyama, Y., et al. 2010, ApJ, 708, 1168

Tinsley, B. M. 1980, FCPh, 5, 287

Tolstoy, E., Hill, V., \& Tosi, M. 2009, ARA\&A, 47, 371

Tolstoy, E., Irwin, M. J., Cole, A. A., et al. 2001, MNRAS, 327, 918

Tolstoy, E., Irwin, M. J., Helmi, A., et al. 2004, ApJ, 617, 119

VandenBerg, D. A., Bergbusch, P. A., \& Dowler, P. D. 2006, ApJS, 162, 375

Venn, K. A., Irwin, M., Shetrone, M. D., et al. 2004, AJ, 128, 1177

Walker, M. G., Mateo, M., Olszewski, E. W., et al. 2009, ApJ, 704, 1274

Wheeler, J. C., Sneden, C., \& Truran, J. W., Jr. 1989, ARA\&A, 27, 279

White, S. D. M., \& Rees, M. J. 1978, MNRAS, 183, 341

Woosley, S., \& Janka, T. 2005, NatPh, 1, 147

Woosley, S. E., \& Weaver, T. A. 1995, ApJS, 101, 181

Worthey, G. 1994, ApJS, 95, 107

Zinn, R., \& West, M. J. 1984, ApJS, 55, 45 